\title{
Composition and Stand Structure of Tropical Moist Deciduous Forest of Similipal Biosphere Reserve, Orissa, India
}

\author{
R.K. Mishra1, V.P. Upadhyay', P.K. Nayak³, \\ S. Pattanaik ${ }^{3}$ and R.C. Mohanty ${ }^{3}$ \\ ${ }^{1}$ Department of Wildlife and Biodiversity Conservation, \\ North Orissa University, Takatpur, Baripada \\ ${ }^{2}$ Ministry of Environment and Forests, Eastern \\ Regional Office, Chandrasekharpur, Bhubaneswar \\ ${ }^{3}$ Department of Botany, Utkal University, Vani Vihar, Bhubaneswar
}

India

\section{Introduction}

Tropical forests are highly productive, structurally complex, genetically rich renewable genetic resources (Roy et al., 2002). The tropical deforestation contributes to increase in atmospheric $\mathrm{CO}_{2}$ and other gases affecting the climate and biodiversity. Though such type of forests occupy less than $7 \%$ of the land surface, there have the higher distinction of harbouring 50\% of all plant and animal species (Mayers, 1992). The rate of forest loss due to deforestation as reported by Food and Agriculture Organistion [FAO, (2001)] is 15.2 million hectare per year (Data from 1990-2000). Assessment of the plant diversity of forest ecosystems is one of the fundamental goals of ecological research and is essential for providing information on ecosystem function and stability (World Conservation Monitoring Centre [WCMC], 1992; Tilman 2000; Townsend et. al., 2008). It has attracted attention of ecologists because of the growing awareness of its importance on the one hand and the massive depletion on the other (Singh, 2002; Lewis, 2009). Out of sixteen major forest types of India (Champion and Seth, 1968), tropical forests occupy $38 \%$ of the total forest area in India (Dixit, 1997). However, in Orissa forest ecosystems cover about $37.34 \%$ of the State's geographical area and about $7.66 \%$ of country's forests. Large population of the state utilizes various components of the forests for both commercial and subsistence purposes. In the past few decades, heavy human pressure has reduced the forested area in the state resulting in degradation and fragmentation of historically contiguous landscapes posing threats to plant diversity (Murthy et al., 2007). It is now high time to conserve the plant diversity and has the task become a major concern for much of the society and for many governments and government agencies at all levels (Tripathi and Singh, 2009).

The Man and Biosphere Programme launched by United Nations Educational Scientific and Cultural Organisation ([UNESCO], 1971 as cited in Parker, 1984) aims at conserving the floral wealth in protected areas established by the Govt. of India in different states. Similipal Biosphere Reserve (SBR), a northern tropical moist deciduous type of forest (Champion and 
Seth, 1968) situated in the Mayurbhanj district of Orissa has over the years, played important roles in maintaining the climate and livelihood of local communities (Srivastava and Singh, 1997; Rout et al., 2010). The SBR located in Eastern Ghat has distinctly dissimilar to the forests located in Western Ghats of India and Srilanka (Table-1). Table-2 provides a comparative account of floristic richness of some tropical forests. Genera like Ficus, Diospyros, Syzygium, Symplocos, Dalbergia, Glochidion are prominently represented in all these ecosystems as shown in Table-1.

\begin{tabular}{|c|c|c|c|}
\hline \multirow[t]{2}{*}{ Name of genera } & \multicolumn{3}{|c|}{ Number of species } \\
\hline & Western Ghats & Srilanka & Similipal \\
\hline Goniothalamus (Annonaceae) & 3 & 5 & 0 \\
\hline Garcinia (Clusiaceae) & 9 & 6 & 0 \\
\hline Calophyllum (Clusiaceae) & 3 & 9 & 0 \\
\hline Mesua (Clusiaceae) & 1 & 3 & 1 \\
\hline Dipterocarpus (Dipterocarpaceae) & 2 & 4 & 0 \\
\hline Hopea (Dipterocarpaceae) & 8 & 4 & 0 \\
\hline Shorea (Dipterocarpaceae) & 1 & 14 & 1 \\
\hline Stemonoporus (Dipterocarpaceae) & 0 & 22 & 0 \\
\hline Pterospermum (Sterculiaceae) & 6 & 0 & 1 \\
\hline Elaeocarpus (Elaeocarpaceae) & 6 & 7 & 0 \\
\hline Ilex (Aquifoliaceae) & 5 & 3 & 0 \\
\hline Euonymus (Celastraceae) & 5 & 3 & 0 \\
\hline Holigarna (Anacardiaceae) & 3 & 0 & 0 \\
\hline Semecarpus (Anacardiaceae) & 2 & 10 & 1 \\
\hline Dalbergia (Leguminosae) & 4 & 1 & 2 \\
\hline Humboldtia (Leguminosae) & 5 & 1 & 0 \\
\hline Syzygium (Myrtaceae) & 29 & 40 & 2 \\
\hline Memecylon (Melastomaceae) & 9 & 25 & 1 \\
\hline Mastixia (Cornaceae) & 1 & 3 & 0 \\
\hline Canthium (Rubiaceae) & 5 & 4 & 0 \\
\hline Ixora (Rubiaceae) & 6 & 4 & 1 \\
\hline Psychotria (Rubiaceae) & 14 & 13 & 0 \\
\hline Lasianthus (Rubiaceae) & 9 & 9 & 0 \\
\hline Vernonia (Asteraceae) & 4 & 11 & 1 \\
\hline Ardisia (Myrsinaceae) & 6 & 6 & 0 \\
\hline Palaquium (Sapotaceae) & 2 & 9 & 0 \\
\hline Diospyros (Ebenaceae) & 16 & 22 & 5 \\
\hline Symplocos (Symplocaceae) & 21 & 12 & 2 \\
\hline Strobilanthus (Acanthaceae) & 9 & 27 & 0 \\
\hline Myristica (Myristicaceae) & 3 & 3 & 0 \\
\hline Cinnamomum (Lauraceae) & 6 & 8 & 0 \\
\hline Actinodaphne (lauraceae) & 5 & 9 & 0 \\
\hline Litsea (Lauraceae) & 8 & 12 & 0 \\
\hline Cleistanthus (Euphorbiaceae) & 2 & 5 & 1 \\
\hline Drypetes (Euphorbiaceae) & 4 & 1 & 0 \\
\hline Glochidion (Euphorbiaceae) & 10 & 9 & 2 \\
\hline Croton (Euphorbiaceae) & 6 & 2 & 1 \\
\hline Agrostistachys (Euphorbiaceae) & 2 & 3 & 0 \\
\hline Mallotus (Euphorbiaceae) & 5 & 4 & 1 \\
\hline Macaranga (Euphorbiaceae) & 2 & 3 & 1 \\
\hline Aporusa (Euphorbiaceae) & 5 & 3 & 0 \\
\hline Ficus (Moraceae) & 10 & 8 & 8 \\
\hline
\end{tabular}

Table 1. Numbers of species in large woody plant genera confined to the Western Ghats, Srilanka and Similipal biosphere reserve (included under Eastern Ghats). 


\begin{tabular}{|l|c|c|c|l|l|}
\hline Forest locations & $\begin{array}{l}\text { Area } \\
\text { (ha) }\end{array}$ & $\begin{array}{l}\text { Number } \\
\text { of species }\end{array}$ & $\begin{array}{l}\text { Number } \\
\text { of genera }\end{array}$ & $\begin{array}{l}\text { Number } \\
\text { of families }\end{array}$ & $\begin{array}{l}\text { Source } \\
\text { of information }\end{array}$ \\
\hline Jadkal forest & 0.5 & 103 & 85 & 46 & Vasanthraj et al., 2005 \\
\hline $\begin{array}{l}\text { Lowland rain forest, } \\
\text { Sabh, Malaysia }\end{array}$ & 8.0 & 329 & 128 & 52 & $\begin{array}{l}\text { Campbell and } \\
\text { Newbery, 1993 }\end{array}$ \\
\hline $\begin{array}{l}\text { Low land dipterocarp } \\
\text { forest, Danum Valley, } \\
\text { Malaysia }\end{array}$ & 8.0 & 511 & 164 & 59 & Newbery et al., 1999 \\
\hline $\begin{array}{l}\text { Keranga forest, } \\
\text { Sarawak and Brunei }\end{array}$ & - & 637 & - & 60 & Newbery, 1991 \\
\hline Similipal, Orissa, India & 3.6 & 266 & 204 & 76 & Present study \\
\hline
\end{tabular}

Table 2. A comparative account of floristic richness of some tropical forest locations.

The National forest policy in India stipulates $33 \%$ of the total geographical area is to be under forest cover. Large area of fertile forest lands have been converted to other land uses to meet the demand of growing population. In addition opening of the close forests due to deforestation has resulted in increase in soil erosion, landslides, floods and loss of biodiversity and wildlife habitats. At the global level similar situation is reported from Brazil, Malyasia, Indonesia, Africa and Central American countries where loss of wildlife habitat ranges from 40-80\% (Puri, 1995). The tropical dry forest of Coasta Rica (Heinrich and Hurka, 2004) is floristically very rich and diverse compared to the dry forests of Puerto Rico (Hare et al., 1997). Compared to other tropical dry deciduous forests of Eastern Ghats of India (Krishnannkutty et al., 2006) which are under various degrees of anthropogenic pressures, the SBR occupies strong ecological position in terms of species number and diversity. SBR is generally believed to be floristically rich, containing many varieties of plant life forms and medicinal plants as well (Saxena and Brahmam, 1989). Carefully compiled and up-to date information on diversity and distribution status of plant resources is however lacking. Though human-induced pressure, mainly through illegal chainsaw logging and access to non-timber forest products (NTFPs) is on the rise (Rout et al., 2009; Rout et al., 2010), a very few sporadic studies of SBR (Mishra et al., 2006, Mishra et al., 2008; Reddy et al., 2007) has so far been conducted to assess the plant diversity status. The conservation status of the biosphere reserve to be known attempting sustainable management, there should be need of proper documentation of diversity status of various plant life forms and their distribution patterns inside the reserve. Knowledge of floristic composition, structure and distribution of angiospermic plants of this biosphere reserve is critical in this direction.

\section{Materials and methods}

\subsection{Study area}

Similipal Biosphere Reserve (SBR) located between $21^{\circ} 28^{\prime}-22^{\circ} 08^{\prime} \mathrm{N}$ latitude and $86^{\circ} 04^{\prime}$ $86^{\circ} 37^{\prime}$ E longitude is situated in the Mayurbhanj district of Orissa stretching over an area of 5569 sq. km (Fig.1). The vast patch of forest covers of Similipal is one of the megabiodiversity zones of the country with a rich population of flora and fauna. The elevation of valley peaks ranges from $80 \mathrm{~m}$ to $869 \mathrm{~m}$ M.S.L. rolling with pockets of grassy meadows in between and traversed by a number of streams and waterfalls. 


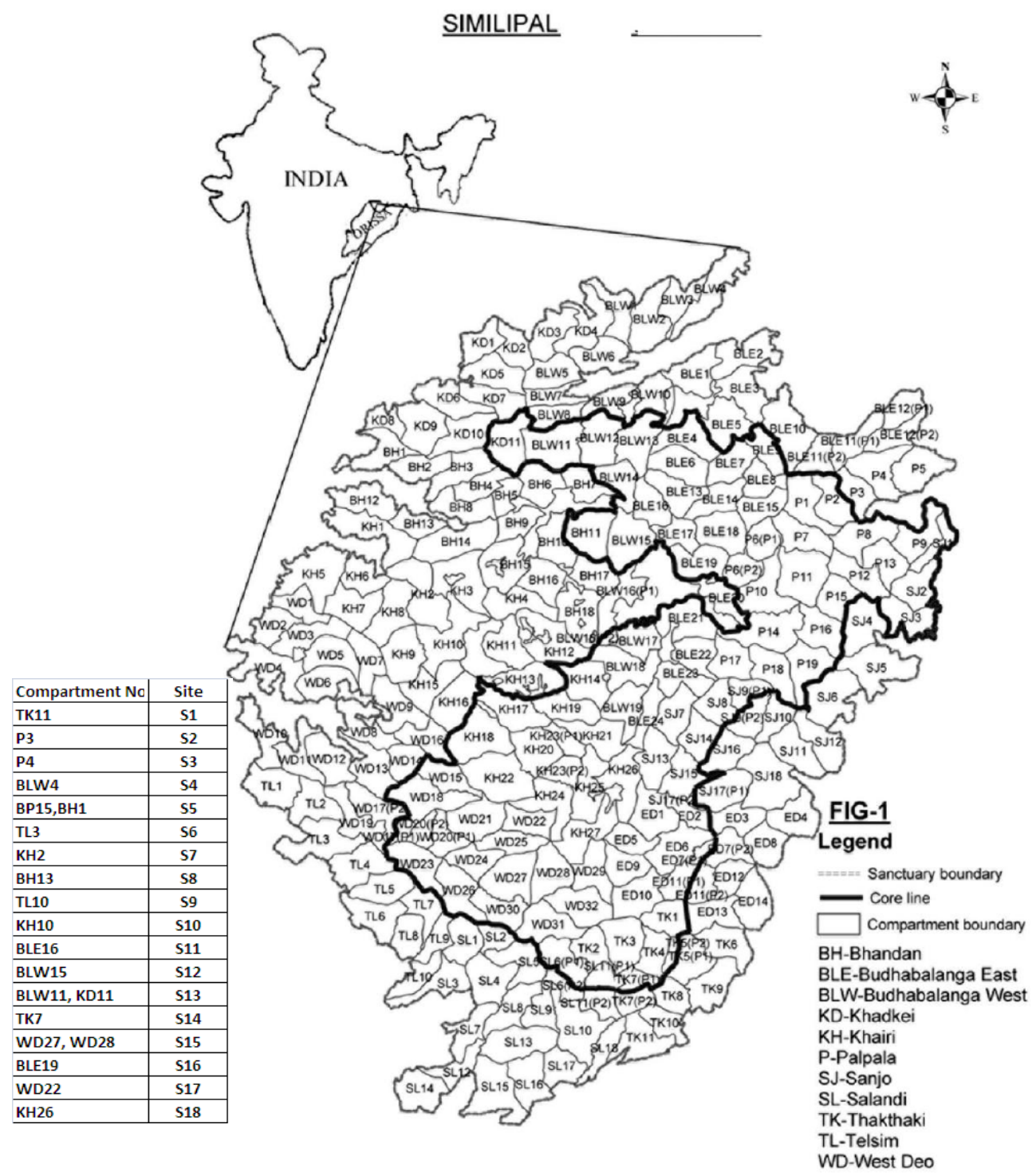

Fig. 1. Location map of sampling sites in Similipal biosphere reserve.

\subsection{Climate}

The climate of the reserve is influenced by a monsoon pattern of rainfall. Maximum rainfall occurs from mid June to October accounting for $75-80 \%$ of annual rainfall. In spite of high annual rainfall summer and winter are relatively dry generally with $<10 \mathrm{~cm}$ monthly rainfall (Mishra et al., 2006). The amount of average annual rainfall is not correlated with elevation and generally ranges from 28.11 to $344.96 \mathrm{~cm}$. Summer is not unbearable, as the maximum 
temperature rarely goes above $40{ }^{\circ} \mathrm{C}$. Winter is severe and the temperature comes down to $4^{0} \mathrm{C}$ in some parts with frosts in valleys (Mishra et al., 2006). Spring is very pleasant. Because of good vegetation and a network of perennial streams Similipal is relatively moist throughout the year. Humidity of Similipal at $0600 \mathrm{hrs}$ is around $40 \%$ and at $1800 \mathrm{hrs}$ is around $81 \%$ to $93 \%$ (Srivastava and Singh, 1997).

\subsection{Field methods (vegetation sampling and analysis)}

To study the plant diversity status, 18 study sites were selected in East, West, North and South directions inside SBR (Fig.1).The vegetation analysis was conducted during 2005-2008 for all the six layers of the forest i.e. trees, climbers, shrubs, herbs, saplings and seedlings. The species were identified with flora guides (Saxena and Brahmam, 1994-1996; Haines, 1921-25). The tree layer was analyzed by sampling 20 quadrats of $10 \mathrm{~m} \times 10 \mathrm{~m}$ size at each site. The size and number of samples were determined using the method of Kershaw (1973) and Mueller-Dombois and Ellenberg (1974). The abundance, density and frequency were calculated for the species. Importance Value Index (IVI) was determined as the sum of the relative frequency, relative density and relative dominance for tree layer only. Raunkiaer's frequency class (1934) analysis was used to assess the rarity or commonness of the tree species (Hewit and Kellman, 2002). In this classification the percentage frequency of the species was classed as A, B, C, D and E; where A represents rare $(0-20 \%), B$ is low frequency $(20-40 \%), C$ is intermediate frequency $(40-60 \%)$, D is moderately high frequency $(60-80 \%)$ and $\mathrm{E}$ is high frequency or common $(80-100 \%)$. With this classification, the expected distribution of the species is $A>B>C \leq \geq D<E$. The distribution pattern of different species was studied using the ratio of abundance to frequency (Whitford, 1949). Trees were $\geq 30 \mathrm{~cm}$ cbh (circumference at breast height), saplings were $10-30 \mathrm{~cm}$ cbh and seedlings were $<10 \mathrm{~cm}$ cbh (Knight, 1975). The shrub and herb layers were analyzed by randomly placing 20 quadrats of $5 \mathrm{~m} \times 5 \mathrm{~m}$ size and $1 \mathrm{~m} \times 1 \mathrm{~m}$ size, respectively at each site during the post monsoon season. The diversity index at each site was computed by using Shannon- Wiener information function (Shannon-Wiener, 1963) and concentration of dominance by Simpson's index (Simpson, 1949), evenness and richness index following Pielou (1975) and Margalef (1958) (as cited in Tripathi and Singh, 2009), respectively. The presence of climbers on trees affects their growth and development. They have been noted to suppress natural regeneration and delay forest recovery (Babweteera et. al., 2001). The presence or absence of climbers on the trees was scored on a 5-point scale (Alder and Synnott, 1992) whereby 1, 2, 3,4 and 5 represented trees that were: having bore climbers; trees over grown with climbers; climbers on the stem only; climbers in the crown only and climbers both on the stem and crown, respectively.

\section{Results}

\subsection{Floristic composition and occurrence}

A total of 266 species belonging to 204 genera and 76 families were recorded from the study area, out of which 117 were tree species, 17 climber, 31 shrub and 101 herb species. Thus only approximately $24.72 \%$ of the estimated flora of Similipal (Saxena and Brahmam, 1989) was covered in the study (Table-3, 4, 5 and 6). A majority of the families were represented by only two or less species. The most common families were Euphorbiaceae and Rubiaceae, each 
represented by 19 species; followed by Fabaceae (15 species), Mimosaceae $=$ Acanthaceae $(12$ species each), Asteraceae (11 species), Cyperaceae $=$ Moraceae $=$ Caesalpinaceae $=$ Combretaceae $(9$ species each $)$, Malvaceae $=$ Melastomataceae $=$ Rutaceae $=$ Poaceae $(7$ species each), etc. The average number of species per hectare was 74 . The number of species per genus was 1.3 and that per family was 3.5. Species in various groups of plant life forms had a wide range of occurrence, ranging in frequency from 5- 72\% in herbs, 5-94\% in shrubs, and 5- 100\% in case of trees, climbers, saplings and seedlings (Table-3, 4, 5, 6, 7 and 8).

\begin{tabular}{|c|c|c|c|c|c|c|}
\hline $\begin{array}{l}\text { Name of the } \\
\text { family }\end{array}$ & Name of the plant species & $\begin{array}{c}\text { Density } \\
\text { (Plants/ha) }\end{array}$ & $\begin{array}{c}\text { Basal } \\
\text { Area } \\
\left(\mathrm{m}^{2} / \mathrm{ha}\right)\end{array}$ & $\begin{array}{c}\text { Frequency } \\
(\%)\end{array}$ & IVI & $\mathrm{A} / \mathrm{F}$ \\
\hline Rubiaceae & $\begin{array}{l}\text { Adina cordifolia } \\
\text { (Roxb.) Hook. f.ex. Brandis }\end{array}$ & 11.94 & 1.44 & 77.78 & 6.54 & 0.04 \\
\hline Rutaceae & Aegle marmelos (L.) Corr. & 4.72 & 0.353 & 44.44 & 2.81 & 0.05 \\
\hline Mimosaceae & Albizia marginata (Lam.) Merr. & 3.33 & 0.37 & 22.22 & 1.80 & 0.14 \\
\hline Combretaceae & $\begin{array}{l}\text { Anogeissus latifolia } \\
\text { (Roxb. ex DC.) Wall ex. Guill }\end{array}$ & 45.28 & 3.36 & 77.78 & 13.43 & 0.15 \\
\hline Bombacaceae & Bombax ceiba L. & 10.83 & 1.53 & 55.56 & 5.64 & 0.07 \\
\hline Euphorbiaceae & Bridelia retusa (L.) Spreng. & 4.72 & 0.45 & 38.89 & 2.73 & 0.06 \\
\hline Anacardiaceae & Buchanania lanzan Spreng. & 19.44 & 0.81 & 66.67 & 6.16 & 0.09 \\
\hline Lecythidaceae & Careya arborea Roxb. & 6.67 & 0.36 & 44.44 & 3.07 & 0.07 \\
\hline Flacourtiaceae & Casearia graveolens Dalz. & 5.28 & 0.12 & 55.56 & 2.98 & 0.03 \\
\hline Caesalpiniaceae & Cassia fistula L. & 6.67 & 0.12 & 44.44 & 3.06 & 0.07 \\
\hline Euphorbiaceae & $\begin{array}{l}\text { Cleistanthus collinus } \\
\text { (Roxb.) Benth. ex Hook.f. }\end{array}$ & 3.33 & 0.06 & 11.11 & 0.94 & 0.54 \\
\hline Euphorbiaceae & Croton roxburghii Balak & 6.11 & 0.21 & 22.22 & 1.92 & 0.25 \\
\hline Mimosaceae & Dalbergia latifolia Roxb. & 2.78 & 0.21 & 22.22 & 1.41 & 0.11 \\
\hline Fabaceae & $\begin{array}{l}\text { Desmodium oojeinesis } \\
\text { (Roxb.) Ohashi }\end{array}$ & 5.00 & 0.58 & 27.78 & 2.53 & 0.13 \\
\hline Dilleniaceae & Dillenia pentagyna Roxb. & 29.17 & 2.53 & 77.78 & 10.24 & 0.10 \\
\hline Ebenaceae & Diospyros embryopteris Pers. & 2.78 & 0.17 & 22.22 & 1.44 & 0.11 \\
\hline Ebenaceae & Diospyros melanoxylon Roxb. & 8.61 & 0.66 & 44.44 & 3.73 & 0.09 \\
\hline Ebenaceae & Diospyros montana Roxb. & 2.78 & 0.07 & 22.22 & 1.34 & 0.11 \\
\hline Burseraceae & Garuga pinata Roxb. & 2.78 & 0.16 & 27.78 & 1.65 & 0.07 \\
\hline Simaroubaceae & Gmelina arborea Roxb. & 5.28 & 0.44 & 50.00 & 3.21 & 0.04 \\
\hline Apocynaceae & $\begin{array}{l}\text { Holarrhena antidysenterica } \\
\text { Wall.ex A.DC. }\end{array}$ & 2.78 & 0.07 & 22.22 & 1.31 & 0.11 \\
\hline Malvaceae & Kydia calycina Roxb. & 5.56 & 0.22 & 38.89 & 2.51 & 0.07 \\
\hline Lythraceae & Lagerstroemia parviflora Roxb. & 6.39 & 0.39 & 33.33 & 2.65 & 0.12 \\
\hline Anacardiaceae & $\begin{array}{l}\text { Lannea corromandelica (Houtt.) } \\
\text { Merr. }\end{array}$ & 4.72 & 0.84 & 27.78 & 2.85 & 0.12 \\
\hline
\end{tabular}


Composition and Stand Structure of

Tropical Moist Deciduous Forest of Similipal Biosphere Reserve, Orissa, India

\begin{tabular}{|c|c|c|c|c|c|c|}
\hline $\begin{array}{l}\text { Name of the } \\
\text { family }\end{array}$ & Name of the plant species & $\begin{array}{c}\text { Density } \\
\text { (Plants/ha) }\end{array}$ & $\begin{array}{c}\text { Basal } \\
\text { Area } \\
\left(\mathrm{m}^{2} / \mathrm{ha}\right)\end{array}$ & $\begin{array}{c}\text { Frequency } \\
(\%)\end{array}$ & IVI & $\mathrm{A} / \mathrm{F}$ \\
\hline Sapotaceae & Madhuca latifolia Gmel. & 12.78 & 1.074 & 44.44 & 4.84 & 0.13 \\
\hline Anacardiaceae & Mangifera indica L. & 3.61 & 1.08 & 38.89 & 3.48 & 0.05 \\
\hline Magnoliaceae & Michelia champaca L. & 7.78 & 0.98 & 11.11 & 2.78 & 1.26 \\
\hline Oleaceae & Nyctanthes arbor-tristis L. & 3.89 & 0.15 & 27.78 & 1.78 & 0.10 \\
\hline Ochnaceae & Ochna obtusata DC. & 5.83 & 0.35 & 16.67 & 1.87 & 0.42 \\
\hline Fabaceae & Pterocarpus marsupium Roxb. & 12.22 & 1.24 & 55.56 & 5.43 & 0.08 \\
\hline Euphorbiaceae & Phyllanthus emblica L. & 4.17 & 0.2 & 27.78 & 1.88 & 0.11 \\
\hline Burseraceae & $\begin{array}{l}\text { Protium serratum } \\
\text { (Wall. ex Colebr.) Engl. }\end{array}$ & 32.22 & 1.99 & 83.33 & 10.08 & 0.09 \\
\hline Mimosaceae & Samanea saman (Jacq.) Merr. & 2.78 & 0.13 & 3.13 & 1.40 & 0.80 \\
\hline Sapindaceae & Schleichera oleosa (Lour.) Oken & 13.33 & 1.48 & 33.33 & 5.05 & 0.24 \\
\hline Euphorbiaceae & $\begin{array}{l}\text { Securinega virosa } \\
\text { (Roxb. ex Willd.) Baill }\end{array}$ & 11.11 & 1.02 & 33.33 & 4.13 & 0.20 \\
\hline \begin{tabular}{|l} 
Diptero \\
carpaceae
\end{tabular} & Shorea robusta Gaertn.f. & 284.17 & 27.73 & 100.00 & 77.67 & 0.57 \\
\hline Myrtaceae & Syzygium cumini (L.) Skeels & 23.06 & 2.03 & 83.33 & 10.19 & 0.07 \\
\hline Myrtaceae & $\begin{array}{l}\text { Syzygium cerasoides } \\
\text { (Roxb.)Chatt. \& Kanjlal }\end{array}$ & 18.06 & 1.27 & 66.67 & 6.64 & 0.08 \\
\hline Combretaceae & $\begin{array}{l}\text { Terminalia alata Heyne ex } \\
\text { Roth. }\end{array}$ & 50.28 & 4.37 & 94.44 & 16.13 & 0.11 \\
\hline Combretaceae & $\begin{array}{l}\text { Terminalia bellirica } \\
\text { (Gaertn.) Roxb. }\end{array}$ & 6.67 & 0.44 & 44.44 & 3.49 & 0.07 \\
\hline Combretaceae & Terminalia chebula Retz. & 5.56 & 0.78 & 61.11 & 4.29 & 0.03 \\
\hline Verbenaceae & Vitex leucoxylon (L.f.) & 5.56 & 0.21 & 38.89 & 2.50 & 0.07 \\
\hline Rubiaceae & $\begin{array}{l}\text { Wendlandia tinctoria } \\
\text { (Roxb.) DC. }\end{array}$ & 3.61 & 0.21 & 27.78 & 1.82 & 0.09 \\
\hline Mimosaceae & Xylia xylocarpa (Roxb.) Taub. & 2.78 & 0.2 & 16.67 & 1.27 & 0.20 \\
\hline Rhamnaceae & Ziziphus mauritiana Lam. & 0.28 & 0.04 & 5.56 & 0.31 & 0.18 \\
\hline Rhamnaceae & Ziziphus rugosa Lam. & 2.78 & 0.05 & 27.78 & 1.48 & 0.07 \\
\hline Total & & 793.67 & 71.043 & - & 299.75 & - \\
\hline
\end{tabular}

Table 3. Families, species, density, basal area, frequency, distribution pattern and Importance Value Index (IVI) of trees in Similipal biosphere reserve. 


\begin{tabular}{|c|c|c|c|c|c|}
\hline $\begin{array}{l}\text { Name of the } \\
\text { family }\end{array}$ & Name of the plant species & \begin{tabular}{|c|} 
Density \\
(Individuals/ha)
\end{tabular} & \begin{tabular}{|c|} 
Frequency \\
$\%$
\end{tabular} & Abundance & $\mathrm{A} / \mathrm{F}$ \\
\hline Euphorbiaceae & Antidesma ghaesembila Gaertn. & 97.78 & 94.44 & 5.18 & 0.05 \\
\hline Myrsinaceae & Ardisia solanacea Roxb. & 45.56 & 55.56 & 4.10 & 0.07 \\
\hline Violaceae & Bixa orellana L. & 17.78 & 22.22 & 4.00 & 0.18 \\
\hline Rubiaceae & $\begin{array}{l}\text { Catunaregam spinosa } \\
\text { (Thunb.) Tirveng. }\end{array}$ & 12.22 & 16.67 & 3.67 & 0.22 \\
\hline Meliaceae & Cipadessa baccifera (Roxb.) Miq. & 3.33 & 5.56 & 3.00 & 0.54 \\
\hline Rutaceae & Citrus medica L. & 48.89 & 44.44 & 5.50 & 0.12 \\
\hline Rutaceae & Clausena excavata Burm. f. & 3.33 & 5.56 & 3.00 & 0.54 \\
\hline Verbenaceae & $\begin{array}{l}\text { Clerodendrum serratum } \\
\text { (L.) Moon }\end{array}$ & 32.22 & 22.22 & 7.25 & 0.33 \\
\hline Euphorbiaceae & Croton caudatus Geisel. & 20.00 & 27.78 & 3.60 & 0.13 \\
\hline Rubiaceae & Gardenia resinifera Roth & 17.78 & 27.78 & 3.20 & 0.12 \\
\hline Euphorbiaceae & Glochidion sp. & 15.56 & 22.22 & 3.50 & 0.16 \\
\hline Lamiaceae & $\begin{array}{l}\text { Gomphostemma parviflorum } \\
\text { Wall. ex Benth. }\end{array}$ & 21.11 & 27.78 & 3.80 & 0.14 \\
\hline Tiliaceae & Grewia hirsuta Vahl. & 22.22 & 27.78 & 4.00 & 0.14 \\
\hline Sterculiaceae & Helicteres isora $\mathrm{L}$. & 17.78 & 22.22 & 4.00 & 0.18 \\
\hline Euphorbiaceae & Homonoia riparia Lour. & 22.22 & 44.44 & 2.50 & 0.06 \\
\hline Hypericaceae & Hypericum gaitii Haines & 54.44 & 22.22 & 12.25 & 0.55 \\
\hline Rubiaceae & $\begin{array}{l}\text { Hyptianthera sticta } \\
\text { (Wild.) Wight \& Arn. }\end{array}$ & 144.44 & 66.67 & 10.83 & 0.16 \\
\hline Fabaceae & $\begin{array}{l}\text { Indigofera cassioides } \\
\text { Rottel ex DC. }\end{array}$ & 125.56 & 50.00 & 12.56 & 0.25 \\
\hline Oleaceae & Jasminum arborescens Roxb. & 10.00 & 27.78 & 1.80 & 0.06 \\
\hline Verbenaceae & Lantana camara L. & 287.78 & 61.11 & 23.55 & 0.39 \\
\hline Vitaceae & Leea asiatica (L.) Ridsdale & 15.56 & 22.22 & 3.50 & 0.16 \\
\hline Vitaceae & Leea indica (Burm. f.) Merr. & 34.44 & 16.67 & 10.33 & 0.62 \\
\hline Melastomataceae & Melastoma malabathricum L. & 122.22 & 50.00 & 12.22 & 0.24 \\
\hline Rubiaceae & Pavetta tomentosa Roxb. Ex Sm. & 22.22 & 27.78 & 4.00 & 0.14 \\
\hline Lamiaceae & $\begin{array}{l}\text { Pogostemon benghalensis } \\
\text { (Burm. f.) Kuntze }\end{array}$ & 341.11 & 55.56 & 30.70 & 0.55 \\
\hline Fabaceae & $\begin{array}{l}\text { Sesbania bispinosa } \\
\text { (Jacq.) W. F. Wight }\end{array}$ & 18.89 & 22.22 & 4.25 & 0.19 \\
\hline Malvaceae & Urena lobata $\mathrm{L}$. & 46.67 & 38.89 & 6.00 & 0.15 \\
\hline Asteraceae & $\begin{array}{l}\text { Vernonia anthelmintica } \\
\text { (L.) Willd. }\end{array}$ & 30.00 & 27.78 & 5.40 & 0.19 \\
\hline Lythraceae & Woodfordia fruticosa (L.) Kurz & 188.89 & 77.78 & 12.14 & 0.16 \\
\hline Rubiaceae & Gardenia gummifera L. f. & 31.11 & 22.22 & 7.00 & 0.32 \\
\hline Fabaceae & $\begin{array}{l}\text { Flemingia chappar } \\
\text { Buch. - Ham. ex Benth. }\end{array}$ & 73.33 & 50.00 & 7.33 & 0.15 \\
\hline Total & & 1944.44 & - & - & - \\
\hline
\end{tabular}

Table 4. Families, species, density, frequency, abundance and distribution pattern of shrub layer in Similipal biosphere reserve. 


\begin{tabular}{|c|c|c|c|c|c|}
\hline $\begin{array}{l}\text { Name of the } \\
\text { family }\end{array}$ & Name of the plant species & \begin{tabular}{|c|} 
Density \\
(Individuals/ha)
\end{tabular} & $\underset{\%}{\text { Frequency }}$ & Abundance & $\mathrm{A} / \mathrm{F}$ \\
\hline Malvaceae & Abutilon indicum (L.) Sweet & 1944.44 & 16.67 & 23.33 & 1.40 \\
\hline Asteraceae & Ageratum conyzoides L. & 1527.78 & 38.89 & 7.86 & 0.20 \\
\hline Acanthaceae & $\begin{array}{l}\text { Andrographis paniculata } \\
\text { (Bum.f.) Wall.ex. Nees }\end{array}$ & 833.33 & 11.11 & 15.00 & 1.35 \\
\hline Commelinaceae & $\begin{array}{l}\text { Aneilema ovalifolium } \\
\text { (Wight) Hook.f.ex. }\end{array}$ & 1472.22 & 11.11 & 26.50 & 2.39 \\
\hline Scrophulariaceae & Bacopa monieri (L.) Pennell. & 2000.00 & 5.56 & 72.00 & 12.96 \\
\hline Capparaceae & Cleome viscosa L. & 1277.78 & 11.11 & 23.00 & 2.07 \\
\hline Commelinaceae & Commelina benghalensis L. & 1083.33 & 11.11 & 19.50 & 1.76 \\
\hline Commelinaceae & Commelina palludosa Bl. & 583.33 & 5.56 & 21.00 & 3.78 \\
\hline Commelinaceae & Commelina sp. & 611.11 & 11.11 & 11.00 & 0.99 \\
\hline Zingiberaceae & Costus speciosus (Koeing) Sm. & 1555.56 & 5.56 & 56.00 & 10.08 \\
\hline Hypoxidaceae & Curculigo orchoides Gaertn. & 6638.89 & 72.22 & 18.38 & 0.25 \\
\hline Zingiberaceae & Curcuma amada Roxb. & 14138.89 & 55.56 & 50.90 & 0.92 \\
\hline Zingiberaceae & Curcuma aromaticum Salisb. & 2111.11 & 27.78 & 15.20 & 0.55 \\
\hline Cyperaceae & Cyperus rotundus L. & 7611.11 & 11.11 & 137.00 & 12.33 \\
\hline Cyperaceae & Cyperus sp. & 1527.78 & 11.11 & 27.50 & 2.48 \\
\hline Fabaceae & Desmodium trifolium (L.) DC. & 35888.89 & 27.78 & 258.40 & 9.30 \\
\hline Acanthaceae & Dicliptera bleupleuroides Mees. & 861.11 & 11.11 & 15.50 & 1.40 \\
\hline Poaceae & Eragrostis cilliata (Roxb.) Nees & 1805.56 & 44.44 & 8.13 & 0.18 \\
\hline Acanthaceae & Eranthemum purpurascens Nees & 916.67 & 22.22 & 8.25 & 0.37 \\
\hline Convolvulaceae & Evolvulus alsinoides L. & 21333.33 & 27.78 & 153.60 & 5.53 \\
\hline Convolvulaceae & Evolvulus numularis (L.) L. & 14694.44 & 11.11 & 264.50 & 23.81 \\
\hline Cyperaceae & $\begin{array}{l}\text { Fimbristylis aestivalis } \\
\text { (Retz.) Vahl. }\end{array}$ & 3138.89 & 11.11 & 56.50 & 5.09 \\
\hline Rubiaceae & Knoxia sumatrensis (Retz.) DC. & 3861.11 & 11.11 & 69.50 & 6.26 \\
\hline Lobeliaceae & Lobelia alsinoides Lam. & 888.89 & 11.11 & 16.00 & 1.44 \\
\hline Cyperaceae & Mariscos sp. & 1500.00 & 11.11 & 27.00 & 2.43 \\
\hline Sterculiaceae & Melotia curcurifolia L. & 777.78 & 5.56 & 28.00 & 5.04 \\
\hline Mimosaceae & Mimosa pudica L. & 611.11 & 5.56 & 22.00 & 3.96 \\
\hline Orobanchaceae & $\begin{array}{l}\text { Orthosiphon rubicundus } \\
\text { (D.Don) Benn. }\end{array}$ & 805.56 & 11.11 & 14.50 & 1.31 \\
\hline Urticaceae & $\begin{array}{l}\text { Pozoulzia pentandra } \\
\text { (Roxb.) Benn. }\end{array}$ & 1111.11 & 16.67 & 13.33 & 0.80 \\
\hline Amaryllidaceae & Pancratium trifolium Roxb. & 1722.22 & 22.22 & 15.50 & 0.70 \\
\hline Acanthaceae & Phlogacanthus sp. & 611.11 & 5.56 & 22.00 & 3.96 \\
\hline Arecaceae & $\begin{array}{l}\text { Phoenix acaulis } \\
\text { Buch-Ham.ex Roxb. }\end{array}$ & 888.89 & 22.22 & 8.00 & 0.36 \\
\hline Arecaceae & Phoenix sp. & 666.67 & 11.11 & 12.00 & 1.08 \\
\hline Euphorbiaceae & Phyllanthus fraternus & 3416.67 & 38.89 & 17.57 & 0.45 \\
\hline Acanthaceae & $\begin{array}{l}\text { Rungia pectinata } \\
\text { (L.) Nees ex DC. }\end{array}$ & 1750.00 & 27.78 & 12.60 & 0.45 \\
\hline Amaranthaceae & Celosia argentia (L.) & 583.33 & 5.56 & 21.00 & 3.78 \\
\hline eae & $\begin{array}{l}\text { Shuteria involucrata } \\
\text { (Wall.) Wt. \& Arn. }\end{array}$ & 583.33 & 27.78 & 4.20 & 0.15 \\
\hline Malvaceae & Sida cordifolia L. & 1111.11 & 11.11 & 20.00 & 1.80 \\
\hline
\end{tabular}




\begin{tabular}{|l|l|c|c|c|c|}
\hline $\begin{array}{l}\text { Name of the } \\
\text { family }\end{array}$ & Name of the plant species & $\begin{array}{c}\text { Density } \\
\text { (Individuals/ha) }\end{array}$ & $\begin{array}{c}\text { Frequency } \\
\%\end{array}$ & Abundance & A/F \\
\hline Rubiaceae & Spermococe pusilla Wall. & 1527.78 & 5.56 & 55.00 & 9.90 \\
\hline Acanthaceae & Strobilanthus auriculatus Nees & 888.89 & 22.22 & 8.00 & 0.36 \\
\hline Fabaceae & $\begin{array}{l}\text { Uraria picta } \\
\text { (Jacq.) Desv. Ex DC. }\end{array}$ & 1138.89 & 16.67 & 13.67 & 0.82 \\
\hline Fabaceae & Zonia dicola & 1166.67 & 11.11 & 21.00 & 1.89 \\
\hline Rubiaceae & Hediotys verticillata (L.) Lam. & 861.11 & 27.78 & 6.20 & 0.22 \\
\hline Orchidaceae & Eulophia nuda Lindi. & 1027.78 & 33.33 & 6.17 & 0.19 \\
\hline Acanthaceae & Barleria srigosa (Wild.) & 611.11 & 38.89 & 3.14 & 0.08 \\
\hline Cyperaceae & Cyperus triceps Endl. & 861.11 & 27.78 & 6.20 & 0.22 \\
\hline Others & Others & 14083.3 & $\begin{array}{c}5.56- \\
27.78\end{array}$ & $2-18$ & $\begin{array}{c}0.06- \\
3.24\end{array}$ \\
\hline Total & & $1,69,500$ & - & - & - \\
\hline
\end{tabular}

Table 5. Families, species, density, frequency, abundance and distribution pattern of herb layer in Similipal biosphere reserve.

\begin{tabular}{|c|c|c|c|c|c|}
\hline $\begin{array}{l}\text { Name of the } \\
\text { family }\end{array}$ & Name of the plant species & \begin{tabular}{|c|} 
Density \\
(Individuals/ha)
\end{tabular} & \begin{tabular}{|c|} 
Frequency \\
$\%$
\end{tabular} & Abundance & $\mathrm{A} / \mathrm{F}$ \\
\hline Liliaceae & Asparagus racemosus Willd & 9.44 & 38.89 & 4.86 & 0.12 \\
\hline Caesalpinaceae & $\begin{array}{l}\text { Bauhinia vahlii } \\
\text { wight. \&Arn }\end{array}$ & 13.33 & 100.00 & 2.67 & 0.03 \\
\hline Fabaceae & Butea superba Roxb & 3.33 & 33.33 & 2.00 & 0.06 \\
\hline Combretaceae & $\begin{array}{l}\text { Calycopteris floribunda } \\
\text { Lam }\end{array}$ & 3.61 & 38.89 & 1.86 & 0.05 \\
\hline Combretaceae & $\begin{array}{l}\text { Combretum roxburghii } \\
\text { Spreng }\end{array}$ & 5.28 & 55.56 & 1.90 & 0.03 \\
\hline Dioscoreaceae & Dioscorea bulbifera L. & 8.06 & 38.89 & 4.14 & 0.11 \\
\hline Mimosaceae & Entada rheedii Spreng & 1.39 & 16.67 & 1.67 & 0.10 \\
\hline Asclepiadaceae & $\begin{array}{l}\text { Hemidesmus indicus } \\
\text { (L.) R.Br. }\end{array}$ & 6.39 & 44.44 & 2.88 & 0.06 \\
\hline Fabaceae & $\begin{array}{l}\text { Millettia extensa } \\
\text { (Benth.) Baker }\end{array}$ & 8.33 & 27.78 & 6.00 & 0.22 \\
\hline Asclepiadaceae & $\begin{array}{l}\text { Pergularia daemia } \\
\text { (Forssk.) Chiov. }\end{array}$ & 13.06 & 44.44 & 5.88 & 0.13 \\
\hline Liliaceae & Smilax macrophylla Roxb & 8.89 & 38.89 & 4.57 & 0.12 \\
\hline Liliaceae & $\begin{array}{l}\text { Smilax prolifera } \\
\text { Wall ex Roxb. }\end{array}$ & 0.56 & 5.56 & 2.00 & 0.36 \\
\hline Apocynaceae & $\begin{array}{l}\text { Aganosma caryophyllata } \\
\text { (Roxb.ex sims)G.Don }\end{array}$ & 1.94 & 16.67 & 2.33 & 0.14 \\
\hline Euphorbiaceae & Bridelia stipularis $\mathrm{Bl}$. & 0.83 & 5.56 & 3.00 & 0.54 \\
\hline Lygodiaceae & $\begin{array}{l}\text { Lygodium flexicosum } \\
\text { (L.) Sw }\end{array}$ & 1.39 & 11.11 & 2.50 & 0.23 \\
\hline Araceae & Pothos scandens L. & 1.94 & 16.67 & 2.33 & 0.14 \\
\hline Oleaceae & Jasminum flexile Vahl & 0.83 & 11.11 & 1.50 & 0.14 \\
\hline Total & & 88.6 & - & - & - \\
\hline
\end{tabular}

Table 6. Families, species, density, frequency, abundance and distribution pattern of climber layer in Similipal biosphere reserve. 
Composition and Stand Structure of

Tropical Moist Deciduous Forest of Similipal Biosphere Reserve, Orissa, India

\begin{tabular}{|c|c|c|c|c|c|}
\hline $\begin{array}{l}\text { Name of the } \\
\text { family }\end{array}$ & Name of the plant species & \begin{tabular}{|c|} 
Density \\
(Individuals/ha)
\end{tabular} & $\begin{array}{c}\text { Frequency } \\
\%\end{array}$ & Abundance & $\mathrm{A} / \mathrm{F}$ \\
\hline Rubiaceae & $\begin{array}{l}\text { Adina cordifolia (Roxb.) } \\
\text { Hook. f.ex. Brandis }\end{array}$ & 18.89 & 44.44 & 2.13 & 0.05 \\
\hline Rutaceae & Aegle marmelos (L.) Corr. & 6.67 & 11.11 & 3.00 & 0.27 \\
\hline Mimosaceae & $\begin{array}{l}\text { Albizia marginata } \\
\text { (Lam.) Merr. }\end{array}$ & 12.22 & 11.11 & 5.50 & 0.50 \\
\hline Combretaceae & $\begin{array}{l}\text { Anogeissus latifolia (Roxb. } \\
\text { ex DC.) Wall ex. Guill }\end{array}$ & 85.56 & 88.89 & 4.81 & 0.05 \\
\hline Barringtoniaceae & $\begin{array}{l}\text { Baringtonia acutangula } \\
\text { (L.) Gaertn. }\end{array}$ & 11.11 & 16.67 & 3.33 & 0.20 \\
\hline Caesalpinaceae & Bauhinia variegata $\mathrm{L}$. & 8.89 & 16.67 & 2.67 & 0.16 \\
\hline Bombacaceae & Bombax ceiba L. & 6.67 & 16.67 & 2.00 & 0.12 \\
\hline Anacardiaceae & Buchanania lanzan Spreng & 58.89 & 55.56 & 5.30 & 0.10 \\
\hline Lecythidaceae & Careya arborea Roxb. & 20.00 & 44.44 & 2.25 & 0.05 \\
\hline Flacourtiaceae & Casearia graveolens Dalz. & 64.44 & 83.33 & 3.87 & 0.05 \\
\hline Caesalpinaceae & Cassia fistula $\mathrm{L}$. & 27.78 & 50.00 & 2.78 & 0.06 \\
\hline Cochlospermaceae & $\begin{array}{l}\text { Chochlospermum } \\
\text { gossypium DC. }\end{array}$ & 7.78 & 5.56 & 7.00 & 1.26 \\
\hline Meliaceae & $\begin{array}{l}\text { Cipadessa baccifera } \\
\text { (Roxb.) Miq. }\end{array}$ & 12.22 & 22.22 & 2.75 & 0.12 \\
\hline Euphorbiaceae & $\begin{array}{l}\text { Cleistanthus collinus } \\
\text { (Roxb.) Benth.-ex Hook. f. }\end{array}$ & 18.89 & 16.67 & 5.67 & 0.34 \\
\hline Euphorbiaceae & Croton roxburghii Balak & 14.44 & 27.78 & 2.60 & 0.09 \\
\hline Fabaceae & $\begin{array}{l}\text { Desmodium oojeinesis } \\
\text { (Roxb.) Ohashi }\end{array}$ & 5.56 & 11.11 & 2.50 & 0.23 \\
\hline Dilleniaceae & Dillenia pentagyna Roxb. & 50.00 & 61.11 & 4.09 & 0.07 \\
\hline Ebenaceae & $\begin{array}{l}\text { Diospyros malabarica } \\
\text { (Desr.) Kostel. }\end{array}$ & 8.89 & 11.11 & 4.00 & 0.36 \\
\hline Ebenaceae & $\begin{array}{l}\text { Diospyros melanoxylon } \\
\text { Roxb. }\end{array}$ & 21.11 & 33.33 & 3.17 & 0.10 \\
\hline Euphorbiaceae & $\begin{array}{l}\text { Glochidion lanceolarium } \\
\text { (Roxb.) Dalz.Glochidion }\end{array}$ & 8.89 & 11.11 & 4.00 & 0.36 \\
\hline Simaroubaceae & Gmelina arborea Roxb. & 8.89 & 22.22 & 2.00 & 0.09 \\
\hline Sterculiaceae & Helicteres isora L. & 10.00 & 22.22 & 2.25 & 0.10 \\
\hline Apocynaceae & $\begin{array}{l}\text { Holarrhena antidysentrica } \\
\text { Wall.ex A.DC. }\end{array}$ & 7.78 & 16.67 & 2.33 & 0.14 \\
\hline Flacourtiaceae & Homalium nepalens Benth. & 40.00 & 61.11 & 3.27 & 0.05 \\
\hline Malvaceae & Kydia calycina Roxb. & 6.67 & 11.11 & 3.00 & 0.27 \\
\hline Anacardiaceae & $\begin{array}{l}\text { Nothopegia heyneana } \\
\text { (Hook. f.) }\end{array}$ & 5.56 & 5.56 & 5.00 & 0.90 \\
\hline Lythraceae & Lagerstroemia parviflora & 17.78 & 27.78 & 3.20 & 0.12 \\
\hline
\end{tabular}




\begin{tabular}{|c|c|c|c|c|c|}
\hline $\begin{array}{l}\text { Name of the } \\
\text { family }\end{array}$ & Name of the plant species & \begin{tabular}{|c|} 
Density \\
(Individuals/ha)
\end{tabular} & $\begin{array}{c}\text { Frequency } \\
\%\end{array}$ & Abundance & $\mathrm{A} / \mathrm{F}$ \\
\hline & Roxb. & & & & \\
\hline Sapotaceae & Madhuca latifolia Gmel. & 13.33 & 27.78 & 2.40 & 0.09 \\
\hline Annonaceae & $\begin{array}{l}\text { Miliusa velutina (Dunal) } \\
\text { Hook.f . \& Thomas. }\end{array}$ & 7.78 & 16.67 & 2.33 & 0.14 \\
\hline Rubiaceae & $\begin{array}{l}\text { Mitragyna parviflora } \\
\text { (Roxb.) Korth. }\end{array}$ & 5.56 & 11.11 & 2.50 & 0.23 \\
\hline Oleaceae & Nyctanthes arber-tristis L. & 36.67 & 72.22 & 2.54 & 0.04 \\
\hline Euphorbiaceae & Phyllanthus emblica L. & 33.33 & 61.11 & 2.73 & 0.04 \\
\hline Burseraceae & $\begin{array}{l}\text { Protium serratum } \\
\text { (Wall. ex Colebr.) Engl. }\end{array}$ & 35.56 & 55.56 & 3.20 & 0.06 \\
\hline Sapindaceae & $\begin{array}{l}\text { Schleichera oleosa } \\
\text { (Lour.) Oken }\end{array}$ & 45.56 & 66.67 & 3.42 & 0.05 \\
\hline Euphorbiaceae & $\begin{array}{l}\text { Securinega virosa } \\
\text { (Roxb. ex Willd.) Baill }\end{array}$ & 38.89 & 50.00 & 3.89 & 0.08 \\
\hline Dipterocarpaceae & Shorea robusta Gaertn.f. & 365.56 & 100.00 & 18.28 & 0.18 \\
\hline Sterculiaceae & Sterculia urens Roxb. & 17.78 & 16.67 & 5.33 & 0.32 \\
\hline Bignoniaceae & $\begin{array}{l}\text { Sterospermum suaveolens } \\
\text { (Roxb.)DC. }\end{array}$ & 13.33 & 16.67 & 4.00 & 0.24 \\
\hline Myrtaceae & $\begin{array}{l}\text { Syzygium cerasoides } \\
\text { (Roxb.)Chatt. \& Kanjlal }\end{array}$ & 18.89 & 33.33 & 2.83 & 0.09 \\
\hline Myrtaceae & $\begin{array}{l}\text { Syzgium cumini } \\
\text { (L.) Skeels }\end{array}$ & 30.00 & 55.56 & 2.70 & 0.05 \\
\hline Combretaceae & $\begin{array}{l}\text { Terminalia alta } \\
\text { Heyne ex Roth. }\end{array}$ & 74.44 & 88.89 & 4.19 & 0.05 \\
\hline Combretaceae & $\begin{array}{l}\text { Terminalia bellirica } \\
\text { (Gaertn.) Roxb. }\end{array}$ & 18.89 & 27.78 & 3.40 & 0.12 \\
\hline Combretaceae & Terminalia chebula Retz. & 11.11 & 33.33 & 1.67 & 0.05 \\
\hline Rubiaceae & $\begin{array}{l}\text { Wendlandia tinctoria } \\
\text { (Roxb.) DC. }\end{array}$ & 20.00 & 33.33 & 3.00 & 0.09 \\
\hline Mimosaceae & $\begin{array}{l}\text { Xylia xylocarpa } \\
\text { (Roxb.) Taub. }\end{array}$ & 15.56 & 27.78 & 2.80 & 0.10 \\
\hline Rhamnaceae & Ziziphus rugosa Lam. & 7.78 & 22.22 & 1.75 & 0.08 \\
\hline Others & & 148.74 & $5.56-16.67$ & $1.00-4.00$ & $0.08-0.72$ \\
\hline Total & & 1524.34 & - & - & - \\
\hline
\end{tabular}

Table 7. Families, species, density, frequency, abundance and distribution pattern of sapling layer in Similipal biosphere reserve. 
Composition and Stand Structure of

Tropical Moist Deciduous Forest of Similipal Biosphere Reserve, Orissa, India

\begin{tabular}{|c|c|c|c|c|c|}
\hline $\begin{array}{l}\text { Name of the } \\
\text { family }\end{array}$ & $\begin{array}{l}\text { Name of the plant } \\
\text { species }\end{array}$ & $\begin{array}{c}\text { Density } \\
\text { (Individuals/ha) }\end{array}$ & \begin{tabular}{|c|} 
Frequency \\
$\%$
\end{tabular} & Abundance & $\mathrm{A} / \mathrm{F}$ \\
\hline Dipterocarpaceae & Shorea robusta Gaertn.f. & 27777.78 & 55.56 & 100.00 & 0.56 \\
\hline Euphorbiaceae & Croton roxburghii Balak & 24250.00 & 145.50 & 33.33 & 4.37 \\
\hline Combretaceae & $\begin{array}{l}\text { Terminalia alata } \\
\text { Heyne ex Roth. }\end{array}$ & 3416.67 & 9.46 & 72.22 & 0.13 \\
\hline Anacardiaceae & $\begin{array}{l}\text { Buchanania lanzan } \\
\text { Spreng. }\end{array}$ & 4944.44 & 14.83 & 66.67 & 0.22 \\
\hline Ebenaceae & $\begin{array}{l}\text { Diospyros melanoxylon } \\
\text { Roxb. }\end{array}$ & 5888.89 & 26.50 & 44.44 & 0.60 \\
\hline Euphorbiaceae & $\begin{array}{l}\text { Cleistanthus collinus } \\
\text { (Roxb.) Benth. Ex } \\
\text { Hook.f. }\end{array}$ & 4611.11 & 23.71 & 38.89 & 0.61 \\
\hline Sterculiaceae & Sterculia urens Roxb. & 1861.11 & 22.33 & 16.67 & 1.34 \\
\hline Flacourtiaceae & $\begin{array}{l}\text { Homalium nepalens } \\
\text { Benth. }\end{array}$ & 1611.11 & 8.29 & 38.89 & 0.21 \\
\hline Euphorbiaceae & Phyllanthus emblica L. & 2138.89 & 12.83 & 33.33 & 0.39 \\
\hline Combretaceae & $\begin{array}{l}\text { Anogeissus latifolia } \\
\text { (Roxb. Ex DC.) Wall ex. } \\
\text { Guill }\end{array}$ & 1500.00 & 13.50 & 22.22 & 0.61 \\
\hline Rutaceae & $\begin{array}{l}\text { Aegle marmelos } \\
\text { (L.) Corr. }\end{array}$ & 972.22 & 7.00 & 27.78 & 0.25 \\
\hline Mimosaceae & Dalbergia sisoo Roxb. & 500.00 & 18.00 & 5.56 & 3.24 \\
\hline Sapindaceae & $\begin{array}{l}\text { Schleichera oleosa } \\
\text { (Lour.) Oken }\end{array}$ & 805.56 & 4.14 & 38.89 & 0.11 \\
\hline Simaroubaceae & Ailanthus sp. Roxb. & 805.56 & 29.00 & 5.56 & 5.22 \\
\hline Apocynaceae & $\begin{array}{l}\text { Hollarhaena } \\
\text { antidysentrica } \\
\text { Wall.ex A.DC. }\end{array}$ & 1361.11 & 7.00 & 38.89 & 0.18 \\
\hline Oleaceae & $\begin{array}{l}\text { Nyctanthes arbortristis } \\
\text { L. }\end{array}$ & 1527.78 & 18.33 & 16.67 & 1.10 \\
\hline Rhamnaceae & Ziziphus rugosa Lam. & 916.67 & 5.50 & 33.33 & 0.17 \\
\hline Euphorbiaceae & $\begin{array}{l}\text { Bridelia retusa } \\
\text { (L.) Spreng. }\end{array}$ & 1277.78 & 11.50 & 22.22 & 0.52 \\
\hline Rubiaceae & Ixora sp. & 722.22 & 26.00 & 5.56 & 4.68 \\
\hline Rubiaceae & Gardenia gummifera .L.f. & 361.11 & 4.33 & 16.67 & 0.26 \\
\hline Fabaceae & $\begin{array}{l}\text { Pterocarpus marsupium } \\
\text { Roxb. }\end{array}$ & 916.67 & 5.50 & 33.33 & 0.17 \\
\hline Fabaceae & $\begin{array}{l}\text { Desmodium oojeinensis } \\
\text { (Roxb.) Ohashi }\end{array}$ & 1194.44 & 10.75 & 22.22 & 0.48 \\
\hline Myrtaceae & $\begin{array}{l}\text { Syzygium cumini } \\
\text { (L.) Skeels }\end{array}$ & 2472.22 & 7.42 & 66.67 & 0.11 \\
\hline Rubiaceae & Wendlandia sp. & 2000.00 & 72.00 & 5.56 & 12.96 \\
\hline Sterculiaceae & Helicteres isora. L. & 972.22 & 8.75 & 22.22 & 0.39 \\
\hline Mimosaceae & Albizia odoratissima. & 1222.22 & 22.00 & 11.11 & 1.98 \\
\hline
\end{tabular}




\begin{tabular}{|c|c|c|c|c|c|}
\hline $\begin{array}{l}\text { Name of the } \\
\text { family }\end{array}$ & $\begin{array}{l}\text { Name of the plant } \\
\text { species }\end{array}$ & $\begin{array}{c}\text { Density } \\
\text { (Individuals/ha) }\end{array}$ & \begin{tabular}{|c|} 
Frequency \\
$\%$
\end{tabular} & Abundance & $\mathrm{A} / \mathrm{F}$ \\
\hline & (L.f.) Benth. & & & & \\
\hline Bignoniaceae & $\begin{array}{l}\text { Sterospermum } \\
\text { suaveolens (Roxb.) DC. }\end{array}$ & 638.89 & 7.67 & 16.67 & 0.46 \\
\hline Combretaceae & $\begin{array}{l}\text { Terminalia chebula } \\
\text { Retz. }\end{array}$ & 861.11 & 5.17 & 33.33 & 0.16 \\
\hline Meliaceae & $\begin{array}{l}\text { Trichilia connaroides } \\
\text { (Wight \& Arn.) Bentv. }\end{array}$ & 638.89 & 23.00 & 5.56 & 4.14 \\
\hline Euphorbiaceae & $\begin{array}{l}\text { Securinega virosa } \\
\text { (Roxb. Ex Wild.) Baill }\end{array}$ & 500.00 & 6.00 & 16.67 & 0.36 \\
\hline Flacourtiaceae & $\begin{array}{l}\text { Casearia graveolens } \\
\text { Dalz. }\end{array}$ & 1750.00 & 6.30 & 55.56 & 0.11 \\
\hline Dilleniaceae & $\begin{array}{l}\text { Dillenia pentagyna } \\
\text { Roxb. }\end{array}$ & 2194.44 & 11.29 & 38.89 & 0.29 \\
\hline Mimosaceae & $\begin{array}{l}\text { Xylia xylocarpa } \\
\text { (Roxb.) DC. }\end{array}$ & 1583.33 & 19.00 & 16.67 & 1.14 \\
\hline Mimosaceae & Dalbergia latifolia Roxb. & 805.56 & 29.00 & 5.56 & 5.22 \\
\hline Burseraceae & $\begin{array}{l}\text { Protium serratum } \\
\text { (Wall.ex Colebr.) Engl. }\end{array}$ & 694.44 & 3.57 & 38.89 & 0.09 \\
\hline Mimosaceae & $\begin{array}{l}\text { Albizia marginata } \\
\text { (Lam.) Merr. }\end{array}$ & 861.11 & 15.50 & 11.11 & 1.40 \\
\hline Rubiaceae & $\begin{array}{l}\text { Adina cordifolia (Roxb.) } \\
\text { Hook. F.ex. Brandis }\end{array}$ & 361.11 & 6.50 & 11.11 & 0.59 \\
\hline Anacardiaceae & Mangifera indica L. & 527.78 & 4.75 & 22.22 & 0.21 \\
\hline Sapotaceae & Madhuca latifolia Gmel. & 583.33 & 10.50 & 11.11 & 0.95 \\
\hline Lecythidaceae & Careya arborea Roxb. & 527.78 & 4.75 & 22.22 & 0.21 \\
\hline Euphorbiaceae & $\begin{array}{l}\text { Securinega virosa } \\
\text { (Roxb. Ex Wild.) Baill }\end{array}$ & 583.33 & 21.00 & 5.56 & 3.78 \\
\hline Simaroubaceae & Gmelina arborea Roxb. & 416.67 & 5.00 & 16.67 & 0.30 \\
\hline Mimosaceae & $\begin{array}{l}\text { Albizia procera } \\
\text { (Roxb.) Benth }\end{array}$ & 388.89 & 14.00 & 5.56 & 2.52 \\
\hline Bombacaceae & Bombax ceiba L. & 388.89 & 7.00 & 11.11 & 0.63 \\
\hline Euphorbiaceae & $\begin{array}{l}\text { Mallotus phillipensis } \\
\text { (Lam.) Muell.-Arg. }\end{array}$ & 416.67 & 15.00 & 5.56 & 2.70 \\
\hline Lythraceae & $\begin{array}{l}\text { Lagerostroemia parviflora } \\
\text { Roxb. }\end{array}$ & 333.33 & 12.00 & 5.56 & 2.16 \\
\hline Rubiaceae & $\begin{array}{l}\text { Wendlandia tinctoria } \\
\text { (Roxb.) DC. }\end{array}$ & 416.67 & 15.00 & 5.56 & 2.70 \\
\hline \multirow[t]{2}{*}{ Meliaceae } & $\begin{array}{l}\text { Cipadessa } \\
\text { baccifera(Roxb.) Miq }\end{array}$ & 333.33 & 12.00 & 5.56 & 2.16 \\
\hline & & 1444.44 & $1.00-9.00$ & $5.56-11.11$ & $0.18-1.62$ \\
\hline Total & & $1,13,416.7$ & - & - & - \\
\hline
\end{tabular}

Table 8. Families, species, density, frequency, abundance and distribution pattern of seedling layer in Similipal biosphere reserve. 
In most of the plant life forms there were a high number of species that occurred only once. The distribution of the species into Raunkiaer's frequency classes showed that most of the species encountered were rare and very few species were in intermediate and high or common frequency class (Table- 9). Except climbers all other groups of plant life forms do not follow the expected $\mathrm{A}>\mathrm{B}>\mathrm{C} \geq \leq \mathrm{D}<\mathrm{E}$ frequency distribution proposed by Raunkiaer (1934) as the number of species in frequency class D is higher than frequency class $E$.

\begin{tabular}{|c|c|c|c|c|c|c|c|c|}
\hline $\begin{array}{c}\text { Frequency } \\
\text { Class }\end{array}$ & Code & \multicolumn{6}{|c|}{ Number of species in vegetation layers } \\
\hline & & Tree & Climber & Shrub & Herb & sapling & seedling & Remark \\
\hline $0-20$ & A & $71(61)$ & $7(41)$ & $4(13)$ & $78(77)$ & $79(73)$ & $34(59)$ & Rare \\
\hline $21-40$ & B & $27(23)$ & $6(35)$ & $16(52)$ & $20(20)$ & $13(12)$ & $18(31)$ & Low \\
\hline $41-60$ & C & $9(08)$ & $3(18)$ & $7(22)$ & $2(02)$ & $7(06)$ & $2(03)$ & $\begin{array}{c}\text { Intermediate } \\
\text { frequency }\end{array}$ \\
\hline $61-80$ & D & $6(05)$ & $0(0)$ & $3(10)$ & $1(01)$ & $5(05)$ & $3(05)$ & $\begin{array}{c}\text { Moderately high } \\
\text { frequency }\end{array}$ \\
\hline $81-100$ & E & $4(03)$ & $1(06)$ & $1(03)$ & $0(0)$ & $4(04)$ & $1(02)$ & $\begin{array}{c}\text { High frequency } \\
\text { (common) }\end{array}$ \\
\hline
\end{tabular}

Table 9. Distribution of vegetation layers according to Raunkiaer's classification scheme (Values in parentheses indicate \% of species).

\subsection{Ecological importance of species}

Importance value Index (IVI) is the measurement of ecological amplitude of species (Ludwig and Reynolds, 1988) suggesting the ability of a species to establish over an array of habitats. However, there is no single perfect way of assessing the ecological amplitude of a species. The abundance of a species can be represented by several measures such as relative density, relative frequency and Importance Value Index (IVI). Though frequency and density values are suitable for herbs and shrubs (Airi et al., 2000), IVI is an important information for tree species. On the basis of IVI, Shorea robusta was found as the dominant species in the SBR having IVI of 77.67 followed by Terminalia alata (16.13) and Anogeissus latifolia (13.43). Wendlandia sp. had IVI of 0.25 and was considered as the rare species of the reserve. All other tree species showed intermediate range of IVI (Table-3).

\subsection{Distribution pattern}

The distribution pattern of trees, shrubs, climbers, herbs, saplings and seedlings of the reserve is shown in Table-10. Odum (1971) stated that under natural conditions, a clumped distribution of plants is normal. A higher percentage of random and regular distribution reflects the greater magnitude of disturbance' such as grazing and lopping in natural forest stands. Most of the species of all the vegetational layers of the reserve showed generally clumped type of distribution in the present study. Regular distribution pattern is completely lacking in all the vegetation layers. Both in herb and seedling layers not a single species showed random distribution pattern (Table-10). 


\begin{tabular}{|c|c|c|c|c|}
\hline Plant group & \multicolumn{4}{|c|}{ Number of species in distribution pattern categories } \\
\hline & Regular & Random & Contiguous & Total number of species \\
\hline Tree & 0 & 08 & 109 & 117 \\
\hline Climber & 0 & 03 & 14 & 17 \\
\hline Shrub & 0 & 01 & 30 & 31 \\
\hline Herb & 0 & 0 & 101 & 101 \\
\hline Sapling & 0 & 06 & 102 & 108 \\
\hline Seedling & 0 & 0 & 58 & 58 \\
\hline
\end{tabular}

Table 10. Distribution pattern of vegetation layers of Similipal biosphere reserve.

\subsection{Distribution of climbers}

Out of 794 number of trees per hectare 110 number of trees per hectare affected by $40 \%$ bore climbers, $10 \%$ were overgrown with climbers while $15 \%$ had climbers restricted to the main stem, $12 \%$ had climbers in the crown only and $23 \%$ had climbers both the stem and in the crown (Figure. 2).

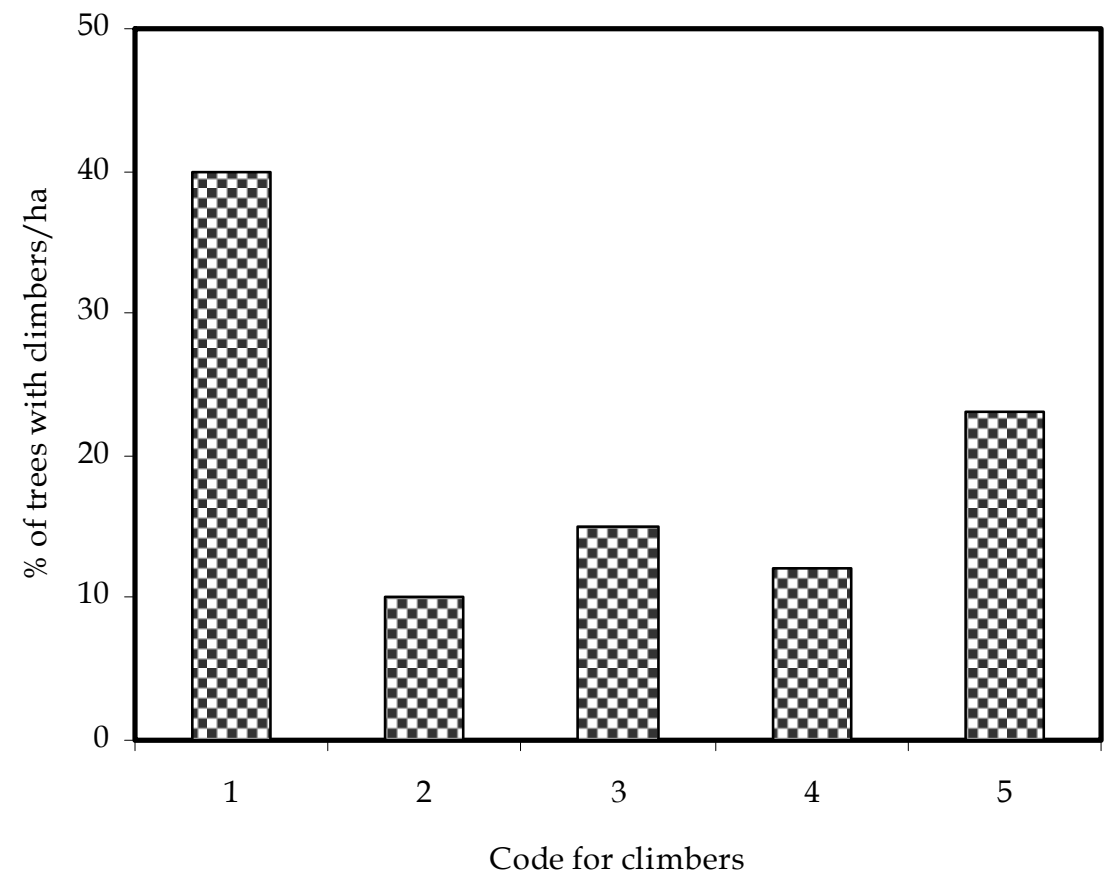

Fig. 2. Percentage distribution of climbers in Similipal biosphere reserve. 


\subsection{Stand structure}

Species wise density of individuals having $\geq 30 \mathrm{~cm}$ girth of the reserve ranged from less than one plant per hectare to 284 plants/ha and the total density of the reserve was 794 plants/ha. Maximum density (per hectare of individuals of $\geq 30 \mathrm{~cm}$ ) was recorded for Shorea robusta (284) followed by Terminalia alata (50), Anogeissus latifolia (45), Protium serratum (32) and Dillenia pentagyna (29). Density was observed less than or equal to one for many species like Antidesma acidum, Artocarpus lacuccha, Butea monosperma,, Casearia elliptica, Chionanthus intermedicus, Cochlospermum religiosum, Euonymus glaber, etc. Some other species showed intermediate range of density per hectare. The densities of climbers in comparison to other vegetation layers of the reserve was too low. However the densities of herbs, seedlings and sapling layers were quite high in comparison to other vegetational layers. Unlike tree layer in herb, shrub and climber layers very few species showed lowest density. Exacum bicolor in herb layer, Cipadesa baccifera and Clausena excavata in shrub layer and, Jasminum flexile and Bridelia stipularis in climber layers values showed minimum value of density (Table-4, 5, 6, 7 and 8). Total basal area of trees of the reserve was $71.05 \mathrm{~m}^{2} /$ ha in which maximum was experienced by Shorea robusta. Shorea robusta contributed maximum of $39 \%$ to the basal area followed by Terminalia alata (6.15\%), Anogeissus latifolia (4.73\%) and Dillenia pentagyna $(3.57 \%)$. The total contribution that resulted from this associated combination of ShoreaTerminalia-Anogeissus-Dillenia was 53.45\%. A few families contributed most to the total basal area. These included Dipterocarpaceae (39\%), Combretaceae (13\%), Myrtaceae (5\%), Rubiaceae (4.5\%) and Moraceae (4\%). As a whole the tree density and basal area of 794 plants/ha and $71.05 \mathrm{~m}^{2} / \mathrm{ha}$, respectively are well within the reported range of various Indian tropical forests (Visalakshi, 1995; Sapkota et al., 2009).

\subsection{Diversity measures}

Species diversity, concentration of dominance and some mathematical indices of different vegetational layers of the reserve are given in Table -11. Measurement of biodiversity of specific area (local scale) on the basis of species richness does not provide a complete understanding about the individuals of the species in an ecosystem as it suffers from the

\begin{tabular}{|c|c|c|c|c|}
\hline \multirow{2}{*}{ Plant group } & \multicolumn{4}{|c|}{ Range of diversity indices } \\
\hline & SD & CD & SR & SE \\
\hline Tree & $1.8-3.11$ & $0.07-0.316$ & $3.36-6.59$ & $0.611-0.951$ \\
\hline Climber & $0.63-1.86$ & $0.155-0.36$ & $0.55-2.22$ & $0.8-0.931$ \\
\hline Shrub & $1.76-2.37$ & $0.102-0.216$ & $1.66-2.92$ & $0.76-0.96$ \\
\hline Herb & $1.57-2.99$ & $0.053-0.323$ & $1.24-4.24$ & $0.63-0.91$ \\
\hline Sapling & $2.1-3.03$ & $0.061-0.194$ & $2.98-6.15$ & $0.816-0.949$ \\
\hline Seedling & $1.01-2.62$ & $0.129-0.397$ & $0.73-4.37$ & $0.61-0.88$ \\
\hline
\end{tabular}

Table 11. Species diversity (SD), Concentration of dominance (CD), species richness (SR) and species evenness (SE) of different forest strata of Similipal biosphere reserve. 
lack of evenness or equitability. It was observed that the richness index ranged from 3.36 to 6.59 (tree layer), 0.55 to 2.22 (climber layer), 1.24 to 4.24 (herb layer), 1.66 to 2.92 (shrub layer), 2.98 to 6.15 (sapling layer) and 0.73 to 4.37 (seedling layer). The equitability showed little variation across the vegetational layers which ranged from 0.61 to 0.95 (tree layer), 0.8 to 0.93 (climber layer), 0.63 to 0.91 (herb layer), 0.76 to 0.96 (shrub layer), 0.82 to 0.95 (sapling layer) and 0.61 to 0.88 (seedling layer). Shannon Wiener's index of diversity is one of the popular measures of species diversity. It ranged from 1.80 to $3.11,0.63$ to $1.86,1.76$ to 2.37 , 1.57 to $2.99,1.01$ to 2.62 and 2.1 to 3.03 for tree, climber, shrub, herb, seedling and sapling layers, respectively, across all sites. Maximum range of species diversity of 1.8 to 3.11 was experienced by tree layer and the minimum range of 0.63 to 1.86 by climber layer indicating that tree layer of SBR was highly diverse while climber layer was the least (Table-11).

\section{Discussion}

\subsection{Floristic composition}

The species richness of a forest ecosystem depends on the number of species per unit area; the more species per unit area, the higher the species richness. A total of 266 species/ 3.6 ha or 74 species/ha in the SBR is more or less similar compared to the number of species reported by several workers in other tropical forest covers of India (Parthasarathy, 1999, 80 to 85 species/ ha in kalakad-MundanthuraiTiger reserve; Parthasarathy and Karthikeyan, 1997, 57 species / ha in Mylodai- Courtallum reserve forest ) and also 70 to 80 species/ha that have been observed in other studies in West African tropical high forests (Lawson, 1985; Vordzogbe et al., 2005). The species richness in neotropical forests showed a wide variation, ranging from 20 species/ha in Varzea forest of Rio Xingu, Brazil (Campbell et al., 1992) to 307 species/ha in the Amazonian Equator (Valenica et al., 1994). In the old world tropics species richness ranged from 26 species/ha in Kolli hills of India (Chittibabu and Parthasarathy, 2000) to 231 species/ha in Brunei Darussalam of South East Asia (Poulsen et al., 1996). In tropical rain forests the range of species count per hectare is about 20 to a maximum of 223. The number of species in SBR was 74 per hectare and this number is at the lower side of the range given in tropical rain forests and neotropical forests. In the study of species richness of the western ghat, south India Sunderpandian and Swamy (2000) stated that pronounced dry season and relatively low annual precipitation factors might be correlated with low species richness.

\subsection{Diversity and related measurements}

It has become common practice in quantitative descriptive studies to use IVI, which combines the relative frequency, density and dominance into a single measure to analyze a plant community. Though vegetation can be described in terms of a number of parameters including frequency, density and cover, the use of any one of these quantitative parameters could lead to over-simplification or under-estimation of the status of the species (Kigomo et. al., 1990, Oyun et. al., 2009). Except few tree species viz. Shorea robusta, Terminalia alata and Anogeissus latifolia, low ecological status of most of the tree species in the present investigation, as evidenced by the IVIs, may be attributed to lack of dominance by any one of these species, suggesting positive interactions among the tree species. In other words, 
resource spaces are shared to minimize negative species interactions and plants can obtain resources with relative ease (Tsingalia, 1990). The low IVIs may also imply that most of the tree species in this forest are rare (Pascal and Pellissier, 1996; Oyun et al., 2009), as confirmed by Raunkiaer's frequency distribution of the tree species (Table- 9). The rarity of species may be attributed to the occurrence of abundant sporadic species with low frequency in the stands (Oyun et al., 2009). The high percentage (>70 \%) of rare species observed in various vegetational layers of the reserve confirms the generally acclaimed notion that most of the species in an ecological community are rare, rather than common (Magurran and Henderson, 2003). The range of evenness value and Simpson's diversity index of 0.61-0.96 and 0.053-0.397, respectively in vegetation layers of Similipal implies that most of the species are equitably distributed while very few species showed the degree of dominance (Pascal and Pellissier, 1996). Shannon Wiener species diversity value among vegetational layers of the reserve ranges from 0.63-3.11 indicating that SBR is highly diverse. The species diversity is generally higher for tropical forests, which is reported as 5.06 and 5.40 for young and old stand, respectively (Knight, 1975). For Indian forests the diversity index ranges between 0.83- 4.1 (Visalakshi, 1995). The diversity index of different vegetational layers of SBR is well within the reported range of the forests of Indian sub-continent (Table-11). Higher species diversity index in tropical forests as reported by Knight (1975) in comparison to the present investigation may be due to differences in the area sampled and lack of uniform plot dimensions. On the other hand, the value obtained for the concentration of dominance for vegetation layers of SBR (0.053-0.397) is greater than those recorded in Nelliampathy (0.085; Chandrashekara and Ramakrishnan, 1994) and tropical dry deciduous forests of Western India (0.08- 0.16; Nirmal Kumar et al., 2010). The high dominance value in the present study indicates single species dominance by Shorea robusta in tree, sapling and seedling layers of the reserve (Table-3, 7 and 8).

\subsection{Distribution pattern of climbers}

The distribution of climbers on the trees of the reserve was considerably low, being nearly equal to $14 \%$. This may be due to high canopy coverage, thereby allowing low light to reach the forest floor and not triggering vigorous growth of climbers (Babweteera et al., 2001). The impact of climbers on the vitality of trees is negative (Toledo- Aceves and Swaine, 2008) causing loss of foliage and thereby reducing the surface area available for metabolic processes and reproductive potential as well as impeding or obstructing forest succession (Toledo-Aceves and Swaine, 2008). Notwithstanding their negative impacts, climbers form bridges between the forest canopies, thereby facilitating the movement of arboreal animals across the forest. They also protect weaker trees from strong winds (Schnitzer and Bongers, 2002).

\subsection{Stand structure}

Stand structure parameters allow predictions of forest biomass and can provide spatial information on potential determinants of plant species distributions (Couteron et al., 2005). In the present study stand structure relates to the basal area of trees, density of trees, and densities of herbs, shrubs, climbers, saplings and seedlings. The tree basal area of $71.05 \mathrm{~m}^{2} /$ ha is high and comparable to the reported range of various Indian tropical forests 
(Visalakshi, 1995; Sapkota et al., 2009) and slightly higher than the value reported from Monteverde of Costa Rica (62 $\mathrm{m}^{2} / \mathrm{ha}$, Nadkarni et al., 1995). High basal area is a characteristic feature of mature forest stand and serves as a reflection of high performance of the trees. It may also presuppose the development of an extensive root system used efficient nutrient absorption, growth suppressing of subordinate plants as the big trees intercept much of the solar radiation that might otherwise reach the forest floor. Dipterocarpaceae had the highest basal area in the present study, followed by Combretaceae, Myrtaceae, Rubiaceae and Moraceae. These families contain important timber species such as Shorea robusta, Terminalia alata, Anogeissus latifolia, Syzygium cumini, Syzygium cerasoides, Terminalia bellirica, Terminalia chebula, etc. The Barringtoniaceae, Chochlospermaceae, Clusiaceae, Malvaceae, Melastomataceae, Myrsinaceae, Ochnaceae, Rosaceae, Salicaceae, Lauraceae, Rhamnaceae, Sterculiaceae, Symplocaceae, Verbenaceae, Flacourtiaceae, and Rutaceae did not contribute much to the total basal area. In all the stands investigated, Chochlospermaceae, Sapotaceae, Salicaceae, etc. were by one individual each while Barringtoniaceae represented by two individuals and, Clusiaceae and Melastomataceae by three individuals each. This implies that very low contribution of these families to the total basal area may be due to their low numbers. Thus, these families may not be very important in terms of dominance. Species wise density of individuals having $\geq 30 \mathrm{~cm}$ girth of the reserve ranged from less than one plant per hectare to 284 plants/ha and the total density of the reserve was 794 plants/ha. Maximum density (per hectare of individuals of $\geq 30 \mathrm{~cm}$ ) was recorded for Shorea robusta (284) followed by Terminalia alata (50), Anogeissus latifolia (45), Protium serratum (32) and Dillenia pentagyna (29). Density was observed less than or equal to one for many species like Antidesma acidum, Artocarpus lacuccha, Butea monosperma," Casearia elliptica, Chionanthus intermedicus, Cochlospermum religiosum, Euonymus glaber, etc. Some other species showed intermediate range of density per hectare. The tree density of 794 individuals/ha recorded in the present investigation is lower as compared to densities reported from Saddle Peak of North Andaman Islands and Great Andaman Groups (946-1137 trees/ha, Padalia et al.,2004). However, the tree density of SBR is comparable with other tropical forests e.g. Kalkad Western Ghats (575-855 trees/ha, Parthasarathy, 1999), Brazil (420-777 trees/ha, Campbell et al., 1992), seasonally deciduous forest of Central Brazil (734 trees/ha, Felfili et al., 2007), Semideciduous forest of Piracicaba, Brazil (842 trees/ha, Viana and Tabanez, 1996) and Costa Rica (617 trees/ha, Heaney and Proctor, 1990). There appears to be little literature available to compare the herb, shrub, sapling and seedling densities with at the local level. The reported densities of these vegetation layers of the reserve in the present investigation is well comparable to Mishra et al. (2008). The fewer numbers of saplings recorded in relation to seedlings in this study implies that most of the saplings are transiting into young trees. It could also mean that most of the seedlings probably die due to intense competition (Weidelt, 1988) for available resources before they reach the sapling stage. Nevertheless, the totality of saplings and seedlings is colossal and reflects high regeneration potential of the forest (Mishra et al., 2005; Khumbongmayum et al., 2006).

\subsection{Comparative analysis of tree species diversity in various tropical forests}

The tree diversity observed in various tropical forests has also been compared with the findings of the present study in SBR (Table-12). The species diversity in SBR can be comparable with other tropical forests. Species richness and density of tree species of the 
present study (117 species and 794 plants per hectare) is well within the reported range of tropical forests in India and outside India. However, the basal area estimated for tree species in the present investigation is well within the reported range of Indian tropical forests but higher than that of tropical forests found outside India (Table-12). The high basal area of $71 \mathrm{~m}^{2} /$ ha obtained in the present investigation was largely due to the contribution of the dominant tree species of the reserve, maximum by Shorea robusta which alone scored $39 \%\left(27.73 \mathrm{~m}^{2} / \mathrm{ha}\right)$ of basal area.

\begin{tabular}{|l|l|l|l|l|l|l|l|}
\hline Forest and location & $\begin{array}{l}\text { No. of } \\
\text { species }\end{array}$ & $\begin{array}{l}\text { No. of } \\
\text { genera }\end{array}$ & $\begin{array}{l}\text { No. of } \\
\text { families }\end{array}$ & $\begin{array}{l}\text { Density } \\
\text { (Plants/ha) }\end{array}$ & $\begin{array}{l}\text { Basal area } \\
(\mathrm{m} 2 / \mathrm{ha})\end{array}$ & Source \\
\hline \multicolumn{7}{|c|}{ Indian tropical forest } \\
\hline $\begin{array}{l}\text { Moist deciduous } \\
\text { forest, Similipal }\end{array}$ & 117 & 87 & 42 & 793.67 & 71.04 & Present study \\
\hline $\begin{array}{l}\text { Moist deciduous } \\
\text { forest, Andaman }\end{array}$ & 235 & 153 & 73 & 946 & 28.60 & Padalia et al., 2004 \\
\hline $\begin{array}{l}\text { Semi evergreen forest, } \\
\text { Andaman }\end{array}$ & 231 & 153 & 71 & 1027 & 33.76 & Padalia et al., 2004 \\
\hline $\begin{array}{l}\text { Evergreen forest, } \\
\text { Andaman }\end{array}$ & 264 & 176 & 81 & 1137 & 44.28 & Padalia et al., 2004 \\
\hline $\begin{array}{l}\text { Wet evergreen forest, } \\
\text { South western ghat }\end{array}$ & 122 & 89 & 41 & $575-855$ & $61.7-94.64$ & Parthasarathy, 1999 \\
\hline $\begin{array}{l}\text { Neotropical cloud } \\
\text { forest, Monteverde, } \\
\text { Costarica }\end{array}$ & 114 & 83 & 47 & 555 & 62.0 & Nadkarni et al., 1995 \\
\hline $\begin{array}{l}\text { Seasonally deciduous } \\
\text { forest,Iaciara, Brazil }\end{array}$ & 39 & - & - & 734 & 16.73 & Felfili et al, 2007 \\
\hline $\begin{array}{l}\text { Seasonally deciduous } \\
\text { forest, Monte Alegre, } \\
\text { Brazil }\end{array}$ & 56 & - & - & 633 & 19.36 & Nascimento et al., \\
\hline $\begin{array}{l}\text { Semideciduous, } \\
\text { Piracicaba, SP, Brazil }\end{array}$ & 101 & - & - & 842 & 12.53 & $\begin{array}{l}\text { Viana and Tabanez, } \\
1996\end{array}$ \\
\hline $\begin{array}{l}\text { Evergreen rain forest, } \\
\text { Ngovayang } \\
\text { (Cameroon) }\end{array}$ & $99-121$ & - & - & $451-634$ & $28.8-42.1$ & Christelle et al., 2011 \\
\hline $\begin{array}{l}\text { Subtropical forest, } \\
\text { Bagh district, } \\
\text { Kashmir, Pakistan }\end{array}$ & 72 & - & 31 & 344 & 69.31 & Saheen et al., 2011 \\
\hline $\begin{array}{l}\text { African wet tropical } \\
\text { forest }\end{array}$ & $344-494$ & - & - & $371-486$ & $27.8-35.8$ & Chuyong et al., 2011 \\
\hline
\end{tabular}

Table 12. Tree species diversity in various tropical forests. 


\section{Conclusion}

The overall analysis indicates that species rich communities of the moist deciduous tropical forests are not only being reduced in area but they are also becoming species poor and less diverse due to rapid deforestation and forest fragmentation. The community organization is also changing in response to increased anthropogenic disturbance. The study has shown that SBR is highly rich in plant diversity and is one of the treasure houses of good ecological wealth of Eastern ghat, India. The long history of timber exploitation prior to its conversion into a biosphere reserve has resulted in the alteration of structure of the forest whereby most of the tree species were affected by very few individuals. The ecological importance of most of the tree species was also low, which reflected rarity of most of the species. However, the abundance of small trees coupled with the colossal sum of saplings and seedlings reflects a high regeneration potential of the forest.

The forest management issues of SBR could be addressed by collection and analysis of long term ecological data which requires scientific baseline studies. We have covered extensively structural parameter analysis which is helpful to know the present state of ecological health of the ecosystem. But due to the various forms of anthropogenic pressure the habitat is destroyed with for logging, illegal hunting, and other challenges (mining in periphery, etc.). The conservation efforts have not so far yielded desired result. With continued biotic pressure and consequent change in structure and function of ecosystem, the management methodology also needs to be modified developing a Long Term Research Network. Similipal is a globally recognized ecosystem covered under UNESCO's Biosphere Reserve housing wide range of flora and fauna. We need to carry out Research and education activities to create an institutional platform to academicians, researchers and scientists. This ecosystem is under pressure. Continued destruction of old-growth and pristine forests of Similipal with high biodiversity will have a regional impact on social and ecological sustainability.

The over exploitation of natural resources in tropical world for meting the basic needs of food, fodder and shelter of local population has disturbed the landscapes causing rapid depletion of biodiversity. Our research results may be of some help to develop management schemes for conservation of biodiversity of SBR. Lack of data base and structural and functional characters of the ecosystem at regular intervals will not help to develop a longterm strategy for sustainable development. Thus continuous collection of data as per longterm action plan on successional status of species level up external and local pressures on the ecosystem, soil fertility management and linkage between social and ecological processes is needed. The community participation and use of traditional technologies as tools for natural resource management should be integrated to achieve sustainable resource management and ecological rehabilitation.

\section{Acknowledgement}

We are thankful to the Director, Dy. Director and other forest officials of Similipal biosphere reserve for their active cooperation in the field work. Financial assistance from CSIR and DST, New Delhi is greatly acknowledged. 


\section{References}

Airi, S., Rawal, R. S., Dhar, U. and Purohit, A. N. (2000). Assessment of Availability and Habitat Preference of Jatamasi a Critically Endangered Medicinal Plant of West Himalayas. Current Science, Vol.79, pp.1467-1470, ISSN 0011-3891.

Alder, D. and Synnott, T. J. (1992). Permanent Sample Plot Techniques for Mixed Tropical Forest. Tropical Forestry Papers 25, ISBN 085074119, University of Oxford, UK.

Babweteera, F., Plumptre, A. and Obua, J. (2001). Effect of Gap Size and Age on Climber Abundance and Diversity in Budongo Forest Reserve, Uganda. African Journal of Ecology, Vol. 38, pp. 230-237, ISSN 0141-6707.

Campbell, D.G., Stone, J.L. and Rosas, A. Jr. (1992). A Comparison of the Phytosociology and Dynamics of Three Floodplain (Varzea) Forest of Known Ages, Rio Jurua, Western Brazilian Amazon. Botanical Journal of the Linnean Society, Vol. 108, pp. 231-237, ISSN 0024-4074.

Campbell, E. J. F. and Newbery, D. McC. (1993). Ecological Relationships Between Lianas and Trees in lowland Rain Forest in Sabh, East Malaysia. Journal of Tropical Ecology, Vol. 9, pp. 469-490, ISSN 0266-4674.

Champion, H.G. and Seth, S.K. (1968). A Revised Survey of the Forest Types of India. Manager of Publication, ISBN 81-8158-0613, New Delhi.

Chandrashekara, U. M. and Ramakrishnan, P. S. (1994). Vegetation and Gap Dynamics of a Tropical Wet Evergreen Forest in the Western Ghats of Kerala, India. Journal of Tropical Ecology, Vol. 10, pp. 337-354, ISSN 0266-4674.

Chittibabu, C.V. and Parthasarathy, N. ( 2000). Attenuated Tree Species Diversity in Human-Impacted Tropical Evergreen Forest Sites at Kolli Hills, Eastern Ghats, India. Biodiversity and Conservation, Vol. 9, pp. 1493-1519, ISSN 0960-3115.

Christelle, F. G., Doumunge, C., Mc Key, D., Tchouto, P. M. G., Sunderland, T. C. H., Balinga, M. P. B. and Snoke, B. (2011). Tree Diversity and Conservation value of Ngovayang's Lowland Forests, Cameroon. Biodiversity and Conservation, DOI 10.10007/s10531-011-0095-Z, ISSN 0960-3115.

Chuyong, G. B., Kenfack, D., Harms, K. E., Thomas, D. W., Condit, R. and Comita, L. S. (2011). Habitat Specificity and Diversity of Tree Species in an African Wet Tropical Forest. Plant Ecology, Vol. 212, pp. 1363-1374, ISNN 1385-0237.

Couteron, P., Pelissier, R., Nicolini, E. A. and Paget, D. (2005). Predicting Tropical Forest Stand Parameters from Fourier Transform of Very High-Resolution Remotely Sensed Canopy Images. Journal of Applied Ecology, Vol. 42, pp. 1121-1128, ISSN 00218901.

Dixit, A.M. (1997). Ecological Evaluation of Dry Tropical Forest Vegetation: An Approach to Environmental Impact Assessment. Tropical Ecology, Vol. 38, pp. 87-100, ISSN 05643295.

FAO (2001). Food and Agriculture organization. State of The Worlds Forests. ISBN 92-5104590-9, Rome.

Felfili, J., Nascimento, A. R. T., Fagg, C. W. and Meirelles, E. A. (2007). Floristic Composition and Community Structure of a Seasonally Deciduous Forest on Limestone Outcrops in Central Brazil. Revista Brasileria de Botanica, Vol. 30, No.4, pp. 611621, ISSN 0100-8404. 
Haines, H.H. (1925). The Botany of Bihar and Orissa. Vol. I-III. London, Botanical Survey of India, Calcutta (Repn.Edn. 1961).

Hare, M. A., Lantage, D. O., Murphy, P. G. and Checo, H. (1997). Structure and Tree Species Composition in a Subtropical Dry Forest in the Dominican Republic: Comparison with a Dry Forest in Puerto Rico. Tropical Ecology, Vol. 38, No. 1, pp. 1-17, ISSN 0564-3295.

Heaney, A. and Proctor, J. (1990). Preliminary Studies of Forest Structure and Floristics on Volcan Barva, Costa Rica. Journal of Tropical Ecology, Vol. 6, pp. 307-320, ISSN 02664674 .

Heinrich, A. and Hurka, H. (2004). Species Richness and Composition During Sylvigenesis in a Tropical Dry Forest in North Western Costa Rica. Tropical Ecology, Vol.45, No. 1, pp. 43-57, ISSN 0564-3295.

Hewit, N. and Kellman, M. (2002). True Seed Dispersal Among Forest Fragments: Dispersal Ability and Biogeographical Controls. Journal of Biogeography, Vol. 29, No. 3, pp. 351 -363, ISSN 0305-0270.

Kershaw, K.R. (1973). Quantitative and Dynamic Plant Ecology. Edward Arnold Ltd., ISBN 0713120991, London.

Khumbongmayum, A.D., Khan, M. L. and Tripathi, R. S. (2006). Biodiversity Conservation in Sacred Groves of Manipur, Northeast India: Population Structure and Regeneration Status of Woody Species. Biodiversity and Conservation, Vol.15, pp. 2439-2456, ISSN 0960-3115.

Kigmo, B. N., Savill, P. S. and Woodell, S. R. (1990). Forest Composition and Its Regeneration Dynamics: A Case Study of Semi-Deciduous Tropical Forest in Kenya. African Journal of Ecology, Vol. 28, No. 3, pp. 174-187, ISSN 0141-6707.

Knight, D.H. (1975). A Phytosociological Analysis of Species Rich Tropical Forest on Barro Colorado Island, Panama. Ecological Monographs, Vol. 45, pp. 259-289, ISSN 00129615.

Krishnankutty, N., Chandrasekaran, S. and Jeyakumar, G. (2006). Evaluation of Disturbance in a Tropical Dry Deciduous Forest of Alagar Hill (Eastern Ghats), South India. Tropical Ecology, Vol. 47, No. 1, pp. 47-55, ISSN 0564-3295.

Lawson, G. W. (1985). Plant Life in West Africa. Ghana Universities Press, ISBN 9964-3-0118-9, Accra.

Lewis, O. T. (2009). Biodiversity Change and Ecosystem Function in Tropical Forests. Basic and Applied Ecology, Vol.10, No. 2, pp. 97-102, ISSN 1439-1791.

Ludwig, J. A. and Reynolds, J. F. (1988). Statistical Ecology: A Primer on Methods and Computing. Wiley-Interscience, ISBN 10-0471832359, New York.

Magurran, A. E. and Henderson, P. A. (2003). Explaining the Excess of Rare Species in Natural Species Abundance Distributions. Nature, Vol. 422, pp. 714-716, ISSN 00280836.

Mayers, N. (1992). The Primary Source: Tropical Forests and Our Future. Norton and Co., ISBN 978-0-393-30828-0, New York.

Mishra, B. P., Tripathi, O. P. and Laloo, R. C. (2005). Community Characteristics of a Climax Subtropical Humid Forest of Meghalaya and Population Structure of 
Ten Important Tree Species. Tropical Ecology, Vol. 46, pp. 241-251, ISSN 05643295.

Mishra, R. K., Upadhyay, V. P. and Mohanty, R. C. (2008): Vegetation Ecology of the Similipal Biosphere Reserve, Orissa, India. Journal of Applied Ecology and Environmental Research, Vol.6, No.2, pp. 89-99, ISSN 1589-1623.

Mishra, R. K., Upadhyay, V. P., Mohapatra, P. K., Bal, S. and Mohanty, R. C. (2006). Phenology of Species of Moist Deciduous Forest Sites of Similipal Biosphere Reserve. Lyonia, Vol.11, No.1, pp.5-17, ISSN 0888-9619.

Mueller-Dombois, D. and Ellenberg, H. (1974). Aims and Methods of Vegetation Ecology. John Wiley and Sons, ISBN 0471622915, New York.

Murthy, M. S. R., Sudhakar, S., Jha, C. S., Reddy, S., Pujar, G. S. and Roy, P. S. (2007). Vegetation, Land Cover and Phytodiversity Characterisation and Landscape Level Using satellite Remote Sensing and Geographic Information System in Eastern Ghats, India. EPTRI-ENVIS Newsletter, Vol. 13, No. 1, pp. 1-12,ISSN 09742336.

Nadkarni, N. M., Matelson, T.S. and . Haber, W.A. (1995). Structural Characteristics and Floristic Composition of Neotropical Cloud Forest, Monte Verde, Costa Rica. Journal of Tropical Ecology, Vol.11, pp. 481-495, ISSN 0266-4674.

Nascimento, A. R. T., Felfili, J. M. and Meirelles, E. M. L. (2004). Floristica e Estrutura De Um Remanscent De Floresta Estucional Decidual De Encosta no Municipio De Monte Alegre, GO, Brazil. Acta Botanica Brasilica, Vol. 18, pp. 659-699, ISSN 01023306.

Newbery, D. McC. (1991). Floristic Variation Within Kerangas (Heath) Forest: revaluation of Data from Sarawak and Brunei. Vegetatio, Vol. 96, pp. 43-86, ISSN 0042-3106.

Newbery, D. McC., Kennedy, D. N., Petol, G. H., Madani, L. and Ridsdale, C. E. (1999). Primary Forest Dynamics in Lowland Dipterocarp Forest at Danum Valley, Sabh, Malaysia and the Role of Understorey. Philosophical Transactions of Royal Society of London, Series B, 354, pp.1763-1782, ISSN 0080-4622.

Nirmal Kumar, J. I., Kumar, R. N., Bhoi, R. K. and Sajish, P. R. (2010). Tree Species Diversity and Soil Nutrient Status in Three Sites of Tropical Dry Deciduous Forest of Western India. Tropical Ecology, Vol. 51, No. 2, pp. 273-279, ISSN 0564-3295.

Odum, E.P. (1971). Fundamentals of Ecology. W.B. Saunders Co., ISBN 0-943451-25-6, Philadelphia.

Oyun, M. B., Bada, S. O. and Anjah, G. M. (2009). Comparative Analysis of the Floral Composition at the Edge and Interior of Agulii Forest Reserve, Cameroon. Journal of Biological Science, Vol.9, No.5, pp.431-437, ISSN 1727-3048.

Padalia, H., Chauhan, N., Porwal, M.C. and Roy, P. S. (2004). Phytosociological Observations on Tree Species Diversity of Andaman Islands, India. Current Science, Vol. 87, pp. 799-806, ISSN 0011-3891.

Parker, J. S. (1984). UNESCO Documents and Publications in the Field of Information: A Summary Guide. IFLA Journal, Vol. 10, No.3, pp. 251-272, ISSN 0340-0352.

Parthasarathy, N. (1999). Tree Diversity and Distribution in Undisturbed and HumanImpacted Sites of Tropical Wet Evergreen Forest in Southern Western Ghats, India. Biodiversity and Conservation, Vol. 8, pp. 1365-1381, ISSN 0960-3115. 
Parthasarathy, N. and Karthikeyan, R.(1997). Biodiversity and Population Density of Woody Species in a Tropical Evergreen Forest in Courtallum Reserve Forest, Western Ghats, India. Tropical Ecology, Vol. 38, pp. 297-306, ISSN 0564-3295.

Pascal L. P., Pellissier, R. (1996). Structure and Floristic Composition of a Tropical Evergreen Forest in South-West India. Journal of Tropical Ecology, Vol.12, pp. 191- 210, ISSN 0266-4674.

Pielou, E.C. (1975). Ecological Diversity. John Wiley and Sons, ISBN 0471689254, New York.

Poulsen, A.D., Nielsen I.V. and Balslev, H. (1996). A Quantitative Inventory of Trees in One Hectare of Mixed Dipterocarp Forest in Temburong, Brunei Durussalam, In Tropical Rain Forest Research-Current Issues, D.S. Edwards, W.E. Booth and S.C. Choy (Eds.) Kluwer Academic Publishers, ISBN 0-7923-4038-8, Dordrecht, The Netherlands.

Puri, G. S. (1995). Biodiversity and Development of Natural Resources for the $21^{\text {st }}$ Century. Tropical Ecology, Vol. 56, No. 2, pp. 253-255, ISSN 0564-3295.

Raunkiaer, C. (1934). The Life Form of Plants and Statistical Plant Geography. Claredon Press ISBN 9978-40-943-2, Oxford.

Reddy, C. S., Pattanaik, C., Mohapatra, A. and Biswal, A. K. (2007). Phytosociological Observations on Tree Diversity of Tropical Forest of Similipal Biosphere Reserve, Orissa, India. Taiwania, Vol. 52, No.4, pp. 352-359, ISSN 0065-1125.

Rout, S. D., Panda, T. and Mishra, N. (2009). Ethnomedicinal Studies on Some Pteridophytes of Similipal Biosphere Reserve, Orissa, India. International Journal of Medicine and Medical Sciences, Vol.1, No.5, pp. 192-197, ISSN 2006-9723.

Rout, S. D., Panda, S. K., Mishra, N. and Panda, T. (2010). Role of Tribals in Collection of Commercial Non-Timber Forest Products in Mayurbhanj District, Orissa. Journal of Studies of Tribes and Tribals, Vol.8, No. 1, pp. 21-25, ISSN 0972-639X.

Roy, P. S., Dutt, C. B. S. and Joshi, P. K. (2002). Tropical Forest Resource Assessment and Monitoring. Tropical Ecology, Vol. 43, No. 1, pp. 21-37, ISSN 0564-3295.

Sapkota, I.P., Tigabu, M. and Oden, P. C. (2009). Species Diversity and Regeneration of Oldgrowth Seasonally Dry Shorea robusta Forests Following Gap Formation. Journal of Forestry Research, Vol. 20, pp. 7-14, ISSN 1007-662X.

Saxena, H.O. and Brahmam, M. (1989): The Flora of Similipahar (Similipal). Orissa. Regional Research Laboratory, Bhubaneswar.

Saxena, H.O. and Brahmam, M. (1996). The Flora of Orissa. Vol. I-IV, Regional Research Laboratory (CSIR), Bhubaneswar and Orissa Forest Development Corporation Ltd., Bhubaneswar.

Schnitzer, S. A. and Bongers, F. (2002). The Ecology of Lianas and Their Role in Forests. Trends in Ecology and Evolution, Vol.17, No.5, pp. 223-230, ISSN 0169-5347.

Shaheen, H., Qureshi, R. A. and Shinwari, Z. K. (2011). Structural Diversity, Vegetation Dynamics and Antropogenic Impact on Lesser Himalayan Subtropical Forests of Bagh District, Kashmir. Pakistan Journal of Botany, Vol. 43, No. 4, pp. 1861-1866, ISSN 0556-3321.

Shannon, C.E. and Wiener, W. (1963). The Mathematical Theory of Communication. University Press, ISBN 0252725484, Illinois, USA. 
Simpson, E.H. (1949). Measurement of Diversity. Nature, Vol. 163, pp. 688, ISSN 00280836.

Singh, J.S. (2002). The Biodiversity Crisis: A Multifaceted Review. Current Science, Vol. 82, pp. 638-647, ISSN 0011-3891.

Srivastava, S. S. and Singh, L. A. K. (1997). Monitoring of Precipitation and Temperature of Similipal Tiger Reserve, In: Similipal: A National Habitat of Unique Biodiversity, P. C. Tripathy and S. N. Patro (Eds.), 30-40, Orissa Environmental Society, ISBN 81900256-5-1, Bhubaneswar.

Sunderpandian, S.M. and Swamy, P.S. (2000). Forest Ecosystem Structure and Composition Along an Altitudinal Gradient in the Western Ghats, South India. Journal of Tropical Forest Science, Vol.12, No. 1, pp. 104-123, ISSN 0128-1283.

Tilman, D. (2000). Causes, Consequences and Ethics of Biodiversity. Nature, Vol. 405, pp. 208-211, ISSN 0028-0836.

Toledo-Aceves, T. and Swaine, M. D. (2008). Effect of Lianas on Tree Regeneration in Gaps and Forest Understorey in a Tropical Forest in Ghana. Journal of Vegetation Science, Vol. 19, No. 5, pp. 717-728, ISSN 1100-9233.

Townsend, A. R., Asner, G. P. and Cleveland, C. C. (2008). The Biogeochemical Heterogeneity of Tropical Forests. Trends in Ecology and Evolution, Vol. 23, No. 8, pp. 424-431, ISSN 0169-5347.

Tripathi, K. P. and Singh, B. (2009).Species Diversity and Vegetation Structure Across Various Strata in Natural and Plantation Forest in Katerniaghat Wildlife Sanctuary, North India. Tropical Ecology, Vol. 50, No. 1, pp. 191-200, ISSN 05643295.

Tsingalia, M. T. (1990). Habitat Disturbance, Severity and Patterns of Abundance in Kakamega Forest, Western Kenya. African Journal of Ecology, Vol. 28, pp. 213- 226, ISSN 0141-6707.

Valencia, R., Balslev, H. and Mino, P. (1994). High Tree Alpha Diversity in Amazonian Ecuador. Biodiversity and Conservation, Vol. 3, pp. 21-28, ISSN 0960-3115.

Vasanthraj, B. K., Shivaprasad, P. V. and Chandrashekar, K. R. (2005). Studies on the Structure of Jadkal Forest, Udupi District, India. Journal of Tropical Forest Science, Vol. 17, pp. 13-32, ISSN 0128-1283.

Viana, V.M. and Tabanez, A.A.J. (1996). Biology and Conservation of Forest Fragments in the Brazilian Atlantic Moist Forest. In: Forest Patches in Tropical Landscapes, J. Schelas and R. Greenberg (Eds.) 151-167. Island Press, ISBN 1-55963-425-1, Washington.

Visalakshi, N. (1995). Vegetation Analysis of Two Tropical Dry Evergreen Forests in Southern India. Tropical Ecology, Vol. 36, pp. 117-127, ISSN 0564-3295.

Vordzogbe, V. V., Attuquayefio, D. K. and Gbogbo, F. (2005). The Flora and Mammals of Moist Semi-Deciduous Forest Zone in the Sefwi Wiawso District of the Western Region, Ghana. African Journal of Ecology, Vol. 8, pp. 49-64, ISSN 02664674.

WCMC (1992). World Conservation Monitoring Centre. Global Biodiversity: Status of Earth's Living Resources. Chapman and Hall, ISBN 0412-47240-6, London, UK. 
Weidelt, H. J. (1988). On the Diversity of Tree Species in Tropical Rain Forest Eosystems. Plant Research and Development, Vol. 28, pp. 110-125, ISSN 0018-8646.

Whitford, P.B. (1949). Distribution of Woody Plants in Relation to Succession and Clonal Growth. Ecology, Vol. 30, pp. 199-208, ISSN 0012-9658. 


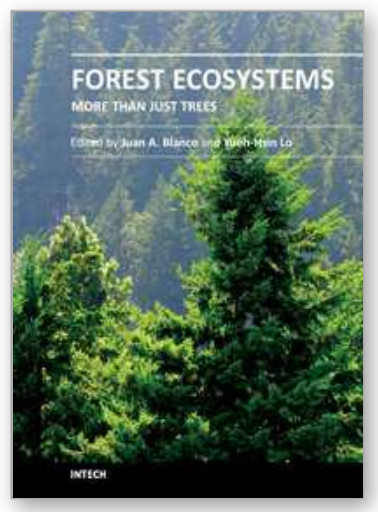

\section{Forest Ecosystems - More than Just Trees}

Edited by Dr Juan A. Blanco

ISBN 978-953-51-0202-1

Hard cover, 464 pages

Publisher InTech

Published online 07, March, 2012

Published in print edition March, 2012

The common idea for many people is that forests are just a collection of trees. However, they are much more than that. They are a complex, functional system of interacting and often interdependent biological, physical, and chemical components, the biological part of which has evolved to perpetuate itself. This complexity produces combinations of climate, soils, trees and plant species unique to each site, resulting in hundreds of different forest types around the world. Logically, trees are an important component for the research in forest ecosystems, but the wide variety of other life forms and abiotic components in most forests means that other elements, such as wildlife or soil nutrients, should also be the focal point in ecological studies and management plans to be carried out in forest ecosystems. In this book, the readers can find the latest research related to forest ecosystems but with a different twist. The research described here is not just on trees and is focused on the other components, structures and functions that are usually overshadowed by the focus on trees, but are equally important to maintain the diversity, function and services provided by forests. The first section of this book explores the structure and biodiversity of forest ecosystems, whereas the second section reviews the research done on ecosystem structure and functioning. The third and last section explores the issues related to forest management as an ecosystem-level activity, all of them from the perspective of the other parts of a forest.

\section{How to reference}

In order to correctly reference this scholarly work, feel free to copy and paste the following:

R.K. Mishra, V.P. Upadhyay, P.K. Nayak, S. Pattanaik and R.C. Mohanty (2012). Composition and Stand Structure of Tropical Moist Deciduous Forest of Similipal Biosphere Reserve, Orissa, India, Forest Ecosystems - More than Just Trees, Dr Juan A. Blanco (Ed.), ISBN: 978-953-51-0202-1, InTech, Available from: http://www.intechopen.com/books/forest-ecosystems-more-than-just-trees/stand-structure-of-tropical-moistdeciduous-forest-of-similipal-biosphere-reserve-orissa-india

\section{INTECH}

open science | open minds

\section{InTech Europe}

University Campus STeP Ri

Slavka Krautzeka 83/A

51000 Rijeka, Croatia

Phone: +385 (51) 770447

\section{InTech China}

Unit 405, Office Block, Hotel Equatorial Shanghai

No.65, Yan An Road (West), Shanghai, 200040, China 中国上海市延安西路65号上海国际贵都大饭店办公楼 405 单元 Phone: +86-21-62489820 
Fax: +385 (51) 686166

Fax: +86-21-62489821

www.intechopen.com 
(C) 2012 The Author(s). Licensee IntechOpen. This is an open access article distributed under the terms of the Creative Commons Attribution 3.0 License, which permits unrestricted use, distribution, and reproduction in any medium, provided the original work is properly cited. 